\title{
La houle à trajectoires fermées en profondeur finie,
}

\section{Closed trajectory waves in a finite depth}

\author{
J. KRAVTCHENKO
}

E T

\author{
A. DAUBERT
}

\begin{abstract}
Mme Dubreil-Jacotin a étendu au cas de la profondeur uniforme finie là solution classique de Gerstner, en démontrant l'existence d'une houle plane telle que toute molécule liquide décrit une trajectoire dans le temps $T$ égal à la période de l'onde. La houle considérée est alors complètement caractérisée par la donnée à priori de son amplitude A.

En utilisant une variante de la méthode de R. Mrche, les auteurs explicitent les équations du phénomène jusqu'au troisième ordre inclusivement par rapport $\dot{a} A$. La définition de ce paramètre à partir des arbitraires de MrCHE a été précisée. Les formules relatives à la distribution du tourbillon, d̀ la célérité et à la forme de la surface libre, sont en accord avec les résultats généraux de Mme Dubneil-Jacotin et de Daubert.

Moyennant certaines hypothèses restrictives, F. Bieser a démontrć que l'absence du transpor de masse (an sens de LEvi-Crvita) caractérisail la houle irrotationnelle; dans un dernier chapitre, les auteurs discutent la portée de ce résultat.
\end{abstract}

\begin{abstract}
Mme Dubrell-Jacotin has extended Gerstner's well-known solution to the uniform finite depth case by demonstrating the existence of a plane wave such that each liquid molecule describes in time $T$ a trajectory that is equal to the period of the wave. The considered wave is thus completely characterised if its amplitude $A$ is given beforehand.

By using an alternative version of Miche's method, the authors explicit the equations of the phenomenon up to and including the 3rd order with respect to $A$. The definition of this parameter, based on Miche's arbitraries, has been precisely stated. The formulae applicable to the distribution of the vortex, the celerity, and the form of the free surface, agree with the general results obtained by Mme DubreilJACOTIN and DAUBERT.

By means of certain restrictive hypotheses, F. BIEser has shown that the absence of mass transport (as understood by Levi-Civita) characterises an irrotational wave. The authors discuss the bearing of this result in the last chapter.
\end{abstract}

\section{I. - INTRODUCTION}

§1. - Madame M.-L. Dubreil-Jacotin a fait connaître dans sa thèse (cf. [1]) $\left(^{*}\right)$ divers théorèmes d'existence pour quelques types de houles d'amplitudes finies. La technique utilisée par cet auteur dans les démonstrations semble avoir limité la diffusion de ces résultats remarquables parmi les techniciens. Nous nous proposons de montrer ici, sur un exemple particulier, le parti qu'on peut tirer du mémoire précité pour le calcul explicite d'un type particulier de houle plane,

(*) Les chiffres entre crochets renvoient à la bibliographie, réunie à la fin de l'article. se propageant dans une couche horizontale, de profondeur constante, d'un liquide parfait, pesant. Nos remarques, d'un niveau très élémentaire, sont loin d'épuiser toutes les applications que l'on peut tirer des travaux de Mme Dubreil, et nous comptons revenir ultérieurement sur ces questions.

$\S 2$. - Rappelons celles des conclusions de [1] qui nous seront directement utiles dans la suite.

Soit $\mathrm{OXY}$, le système d'axes rectangulaires, liés à la houle. Il faut entendre par là que $O X Y$ se déplace, relativement au repère fixe, 
d'un mouvement de translation horizontale et uniforme, de vitesse égale à la célérité de l'onde : par définition, le mouvement relatif du liquide par rapport à $\mathrm{O} X \mathrm{Y}$ est permanent.

Nous prendrons $\mathrm{O} Y$ vertical ascendant, $\mathrm{O} X$ orienté en sens contraire de la propagation el situé dans le niveau moyen.

Appelons : $H$, la profondeur (supposée finie et constante) du liquide au repos; $\lambda$, la longueur d'onde de la houle; $c$, sa célérité; $\mu$, un paramètre positif (assez petit) dont nous préciserons tout à l'heure le rôle; A, l'amplitude de la houle; $\Psi(\mathrm{X}, \mathrm{Y})$, la fonction de courant : $\zeta(\mathrm{X}$, $Y)$ le tourbillon; $q$ le débit relatif du liquide. D'après un résultat classique, les lignes de courant : $\psi=$ Constante, sont isotourbillonnaires et on $a$ :

$$
\zeta=f(\psi)
$$

Yoici les hypothèses que nous ferons relativement à $f$ et $\mu$.

Le paramètre positif y. sera toujours inférieur à $\mu_{0}, \mu_{0}$ étant une fonctionnelle compliquée de $\lambda$, $\mathrm{H}$ et $f$.

La fonction $f(\psi)$, définie pour $0 \leqslant \psi \leqslant q$ sera supposée continue au sens de Hölder sur cet intervalle, extrémités comprises. En d'autres termes, $\psi$ et $\psi$ étant deux valeurs arbitraires de cet intervalle fermé, on a :

$$
\left|f(\psi)-f\left(\psi^{\prime}\right)\right| \leqslant \mathrm{C}\left|\psi-\psi^{\prime}\right|^{\alpha} .
$$

$C$ et $\propto$ étant deux constantes, dont la première n'est pas négative et dont la seconde vérifie les inégalités :

$$
0 \leqslant \alpha \leqslant 1
$$

Une fonction hölderienne quelconque $f(\psi)$ n'est pas, en général, analytique en $\psi$; mais toute fonction analytique régulière pour $0 \leqslant \psi \leqslant q$ est hölderienne sur cet intervalle. Enfin, nous supposerons que $|f(\psi)|$ est assez petit, de l'ordre de $\mu_{0}$, au plus.

\$3. - Cela étant, Mme Dubreil établit le théorème d'existence et d'unicité suivant : à un choix arbitraire de $\lambda$, $u, H$, et $f(\psi)$ correspond une onde progressive, plane et périodique, de période $\lambda$ en $X$, et une seule $\left(^{\star}\right)$. De plus, cet auteur indique plusieurs propriétés de la solution du problème de l'onde : nous allons rappeler celles de ces propriétés qui peuvent être utiles au calculateur, soit pour justifier ses méthodes

$\left(^{\star}\right)$ En fait, Mme Dubneil prend comme donnée la fonction $h(0)$, liée à $f(\psi)$ au moyen des relations :

$$
\begin{gathered}
\rho=e^{-(2 \pi a / \lambda) \psi ;} \\
h(\rho)=-\left(\lambda^{2} / 4 \pi^{2}\right) a f(\psi) / \rho^{2},
\end{gathered}
$$

où $a$ est un paramètre positif, qui est une fonctionnelle très compliquée des données. d'approximation, soit pour le guider dans le choix des inconnues auxiliaires.

L'écoulement possède une infinité d'axes de symétrie verticaux, la distance (horizontale) de deux axes consécutifs étant de $\lambda / 2$; en deux points symétriques par rapport à un axe quelconque de symétrie, les composantes horizontales du vecteur vitesse sont égales, alors que les composantes verticales ont des signes opposés. Le paramètre $\mu$ caractérise l'amplitude A de l'onde, et inversement; les composanles de la vitesse sont des fonctions analyliques de $\mu$ et possèdent des dérivées continues des deux premiers ordres en $\mathrm{X}$ et $\mathrm{Y}$.

L'énoncé d'existence, qui précède, sous-entend que les autres paramètres caractérisant l'écoulement envisagé sont déterminćs à partir des données. En particulier, le débit $q$, qui fixe l'intervalle des variations de $\psi$, se trouve être solution unique d'une équation transcendante. Ia délermination de $c$ mérite une mention spéciale. En fait, on a établi l'existence d'un écoulement permanent en axes mobiles O X Y; les écuations du mouvement seront encore satisfaites dans tout système de référence animé d'un mouvement de translation horizontale et uniforme relativement à OXY. Dès lors, un certain arbitraire intervient dans le choix du système de référence auxiliaire qu'on appellera fixe. Nous renvoyons à $[1], \S 5$, pour la discussion de ce point capital. Mais, quelle que soit la définition adoptée pour $c$, la théorie de Mme Dubreil montre que ce paramètre, essentiel au point de vue physique, est une fonctionnelle compliquée des données, c'està-dire de $\lambda, \mathrm{H}$, $\mathrm{A}$, et $f(\psi)$.

Il ne semble pas que cette conséquence de la théorie ait été assez remarquée. Nous pourrions citer plusieurs mémoires récents consacrés au calcul approché de $c$, en fonction de $\lambda, H$ et $A$ seulement; il semble que les auteurs de ces travaux négligent systématiquement l'influence que peut avoir, à ce point de vue, l'état tourbillonnaire de l'onde. Or, à la suite de [1], nous allons rappeler que $f(\psi)$ influe sensiblement sur $c$. En effet, si $f(\psi)$ est le premier ordre en $\mu$. (c'esta-dire en $\mathrm{A}$ ), on aura :

$$
c^{2}=\operatorname{th} \frac{\lambda g}{2 \pi} \frac{2 \pi \mathrm{H}}{\lambda}+\mathrm{AF}(\mathrm{A}, \lambda, \mathrm{H}, f),
$$

F étant une fonctionnelle compliquée de ses arguments, en général, finie pour $\mathrm{A}=0$; on reconnaît dans le premier terme du second membre la célèbre expression d'Airy, valable dans le cas de la houle linéaire.

Ce n'est que dans des cas exceptionnels, au point de vue analytique, que $F$ est de second ordre en A. Il se trouve, par bonheur, qu'il en est ainsi si $f=0$; c'est le cas de l'onde irrotationnelle de Levi-Civita-STrurck (cf. [2], [3]) 
bien étudié au point de vue numérique par beaucoup d'auteurs dont G. STokes et L. SRETENSKY (Cf. [4]). On sait ainsi que pour la gamme courante des amplitudes, le terme A F, du second ordre en $\mathrm{A}^{2}$ (dans le cas irrotationnel) est pratiquement négligeable vis-à-vis du terme classique d'Airy. Or, dans le cas des expériences de laboratoire, $f(\psi)$ semble avoir souvent une valeur très faible. Cela tient peut-être à ce que l'état initial du liquide est le repos. Si donc, on néglige la viscosité, les mouvements de l'eau, excités par l'appareil générateur de la houle, seront, en vertu du théorème de Lagrange, toujours irrotationnels. Ainsi la formule d'Airy estelle quotidiennement utilisée avec succès dans les laboratoires, et il faut des expériences très fines pour mettre en évidence les effets sur la célérité de la non-linéarité des lois du phénomène. C'est pourquoi, sans doute, les techniciens négligent systématiquement la correction de premier ordre en A à l'expression de la célérité des ondes de gravité. Pourtant, il nous semble qu'il serait très utile de former les expressions approchées explicites des lois d'une houle, correspondant à une répartition tourbillonnaire de premier ordre en A; en dehors de l'intérêt analytique de la question, on aurait, peut-être, ainsi l'explication de quelques anomalies que nous avons observées au cours des expériences de laboratoire. A première vue, le problème semble assez difficile, mais nous espérons revenir sur ce point dans un travail ultérieur.

Depuis que ces lignes ont été écrites, A. DAUBERT a résolu le problème et a explicité l'équation des lignes de courant en axes O X Y, dans le cas d'une houle quelconque caractérisée par une répartition arbitraire $f(\psi)$ du tourbillon. Il en déduit la valeur du terme correctif à la formule d'Airy, en adoptant pour la célérité la définition de F. Bresel (Cf. [8]). Ces résultats sont en cours de publication dans les comptes rendus de l'Académie des Sciences de Paris.

$\S 5$. - L'objet de ce mémoire est plus modeste. Nous nous proposons de calculer au troisième ordre près en A, les caractéristiques d'une onde très remarquable qui généralise (au cas de la profondeur finie) la célèbre houle trochoïdale de Gertsner, dont Mme Dubrerl a établi l'existence. Le régime en cause est caractérisé par cette propriété que la trajectoire absolue de toute molécule du liquide est fermée et décrite en un temps $\lambda / c$; la fonction $f(\psi)$ correspondante est alors entièrement déterminée par la donnée de $\lambda, H$ et $\mu$; elle est du second ordre en $\mu$, ainsi que la quantité : $c^{2}-(\lambda g / 2 \pi)$ th $(2 \pi \mathrm{H} / \lambda)$.

Le transport absolu des masses est nul dans ce cas; il ne l'est pas au sens de Levi-Civita.

$\S 6$. - Disons quelques mots des méthodes que l'on peut mettre en œuvre pour former les solutions approchées du problème de l'onde périodique.

Au $\S 3$ de [1], on trouvera, rapidement indiqué, le principe d'un procédé de calcul, directement lié aux équations de départ de Mme DuBREIL. L'avantage de cette méthode est évident : elle conduit sûrement au but fixé, puisqu'elle repose sur des théorèmes d'existence rigoureux. En revanche, ce procédé exige l'emploi des paramètres et des variables auxiliaires dépourvus de signification physique directe. C'est pourquoi nous avons choisi une voie différente. Nous utilisons une variante (inspirée, d'ailleurs, par les résultats de [1]) du processus d'approximation de R. Miche (cf. [5]), qui semble spécialement bien adapté au cas particulier que nous nous proposons d'étudier. En effet, les paramètres, choisis comme des données arbitraires, ont une interprétation physique immédiate : ce sont la période $\mathrm{T}$ et l'amplitude de l'onde; de plus, en vertu des hypothèses faites quant à la nature de l'onde, les termes séculaires sont nuls dans les approximations de chaque ordre. Il en résulte une grande simplification des calculs, ce qui ne reut pas dire qu'ils deviennent simples!

Toutefois, la justification rigoureuse de notre processus d'approximation exige la démonstration préalable de quelques résultats d'analyse. Les variables que nous utilisons ne sont pas celles de [1]; il est alors indispensable de prouver l'équivalence de notre système de données arbitraires avec celui de [1]. Pour alléger notre exposé, nous renvoyons à une publication ultérieure l'étude de ces points que le lecteur admettra, nous l'espérons du moins, sans peine. L'essentiel de notre calcul a été publié dans [6].

Pour finir, nous présenterons quelques remarques sur la houle irrotationnelle de Levi-Crvita et de Struick; certains résultats de [1] nous permettent de préciser la portée d'un récent travail de M. F. Bieser (cf. [7]).

\section{II. - NOTATIONS ET ÉQUATIONS DU PROBLĖME}

$\S 7$. - Nous rapporterons le mouvement à un système de référence fixe $O x y$; l'axe $O x$ sera orienté dans le sens de la propagation de l'onde et situé dans le plan d'eau au repos; o $y$ sera orienté suivant la verticale ascendante. Nous nous proposons d'étudier dans ce repère une 
houle périodique progressive plane, à trajectoires fermées, décrites dans le même temps.

Les notations utilisées ci-après diffèrent en quelques points de détail de celles du premier chapitre. Nous appellerons encore $: \lambda$, la longueur d'onde de la houle; A, son amplitude; T, sa période; $c$, sa célérité; $\mathrm{H}$, la profondeur (finie et constante) du liquide. Les données arbitraires, caractérisant le phénomène, seront ici $\mathrm{T}$ et $\mathrm{A}$. Le problème revient à exprimer le champ des déplacements et le paramètre $c$ [ou ce qui revient au même, $\lambda=(c / \mathrm{T})$ ], en fonction de $\mathrm{H}$, $\mathrm{A}$ et de $\mathrm{T}$, au moyen des formules approchées, exactes au troisième ordre près en $\mathrm{A}^{3}$.

D'après le résultat de [1], rappelé au $\S 5$, il n'existe qu'un seul type de houle à trajectoires fermées, décrites dans le temps $\mathrm{T}$; la répartition du tourbillon dans le liquide est, dès lors, entièrement déterminée (mais non explicitement connue à priori) dès qu'on se fixe les valeurs de $\mathrm{H}, \mathrm{A}$ et $\mathrm{T}$.

\$ 8. - Ici apparaît une première difficulté d'ordre théorique. Nous ignorons l'intervalle $0<\mathrm{A} \leqslant \mathrm{A}_{0}$, à l'intérieur duquel les résultats de Mme Dubreil, que l'on vient de rappeler, restent valables (cf. § 2). Nous supposerons done simplement que A est petit, sans assigner de borne supérieure à ce paramètre. On ne connaîtra donc pas la gamme des amplitudes pour lesquelles les développements limités ci-après seront légitimes.

§ 9. - Il nous faut maintenant faire un choix judicieux du système de variables indépendantes. Or, nous savons, à priori, que les trajectoires des molécules sont des courbes fermées, dont le diamètre est une fonction analytique de A, s'annulant avec ce paramètre; le mouvement à amplitude nulle se réduit au repos. Il paraît donc naturel de choisir comme variables indépendantes, en dehors du temps $t$, les coordonnées moyennes $x_{0}, y_{0}$ de la molécule au repos. $\mathrm{Si}$, dès lors, les équations paramétriques de la trajectoire absolue d'une molécule individualisée sont de la forme:

$$
\begin{aligned}
& x=x(t, \mathrm{~A}), \\
& y=y(t, \mathrm{~A}),
\end{aligned}
$$

nous sommes amenés à prendre :

$$
\begin{aligned}
& x_{0}=x(t, 0) \\
& y_{0}=y(t, 0)
\end{aligned}
$$

formules dont les seconds membres doivent être indépendants du temps. Mais, il faut bien remarquer que le domaine des variations de $x_{0}, y_{0}$ n'est pas déterminé à priori : la houle de Gertsner fournit un exemple facile de ce fait. On sait que, dans ce cas (où $H=\infty$ ), les équations du mouvement sont données par le système :

$$
\begin{aligned}
& x=\alpha+\mathrm{R} e^{k \beta} \sin (k \alpha-s t), \\
& y=\beta-\mathrm{R} e^{k \beta} \cos (k \alpha-s t),
\end{aligned}
$$

où $\mathrm{R}, k, s$ sont des constantes, dont la première est arbitraire et où $\alpha$ et $\beta$ sont les coordonnées du centre de l'orbite circulaire, décrite par la molécule individualisée au moyen des paramètres $\alpha, \beta$. Si donc $\mathrm{R} \rightarrow 0$, on devrait prendre $x_{0}=\alpha, y_{0}=\beta$. On montre aisément que le point $\alpha, \beta$ décrit un demi-plan lorsque le point $x, y$ balaie le domaine du liquide en mouvement; mais, ce demi-plan est distinct de celui occupé par la masse liquide au repos et dépend du choix de $k$ et de $\mathrm{R}$.

On doit conclure de là que la définition précise de la position moyenne soulève des difficultés sur lesquelles nous reviendrons longuement ailleurs. Notre but actuel est de signaler seulement cette circonstance et de montrer que l'emploi rigoureux des coordonnées moyennes exige des précautions.

Cecị étant, nous admettrons qu'il est possible de paramétrer le mouvement envisagé au moyen des formules du type de Miche :

$$
\begin{aligned}
& x=x_{0}+\mathrm{X}\left(x_{0}, y_{0}, t, \mathrm{~A}\right) ; \\
& y=y_{0}+\mathrm{Y}\left(x_{0}, y_{0}, t, \mathrm{~A}\right),
\end{aligned}
$$

où le point moyen $x_{0}, y_{0}$ balaie toute la bande horizontale $\Delta$ :

$$
\left.\begin{array}{l}
-\infty \leqslant x_{0} \leqslant \infty ; \\
-H \leqslant y_{0} \leqslant 0,
\end{array}\right\}
$$

occupée par le liquide au repos. On voit donc que $\mathrm{X}$ et $\mathrm{Y}$ sont les composantes, à l'époque $t$, du vecteur déplacement de la molécule, individualisée au moyen de ses coordonnées de repos $x_{0}$ et $y_{0}$. D'après [1], $\mathrm{X}$ et $\mathrm{Y}$ sont des fonctions périodiques du temps $t$ (de période $\mathrm{T}$ ); en effet, le vecteur déplacement doit, par hypothèse, prendre la même valeur aux époques $t$ et $\mathrm{KT}+t$, $\mathrm{K}$ étant entier. Nous admettrons que ce sont aussi des fonctions périodiques de $x_{0}$ de période $\lambda$. Si l'on veut représenter au moyen de (1), une onde progressive, nous sommes amenés à postuler que $\mathrm{X}$ et $\mathrm{Y}$ ne dépendent de $x_{\mathrm{f}}$ et de $t$ que par l'intermédiaire de la seule variable auxiliaire :

$$
\left.\begin{array}{l}
v=u x_{0}-\nu t ; \quad-\infty \leqslant v \leqslant \infty \\
\mu=(2 \pi / \lambda) ; \\
\nu=(2 \pi / \mathrm{T}) ;
\end{array}\right\}
$$

relativement à laquelle $\mathrm{X}$ et $\mathrm{Y}$ admettent la 
même période $2 \pi$. Il suffit donc de déterminer $\mathrm{X}$ et $\mathrm{Y}$ dans le rectangle $\mathrm{D}$ :

$$
\begin{aligned}
& -\pi \leqslant v \leqslant \pi ; \\
& -\mathrm{H} \leqslant y_{0} \leqslant 0 ;
\end{aligned}
$$

pour que le vecteur déplacement soit défini dans toute la bande $\Delta$.

Mais d'après [1], le mouvement possède une infinité d'axes verticaux de symétrie dans le plan des variables $v, y_{0}$ balayant le domaine $\Delta$, la distance de deux axes consécutifs valant $\pi$. On peut donc supposer, sans restreindre la généralité, que $\mathrm{X}$ est une fonction paire de $v$, alors que $\mathrm{Y}$ est une fonction impaire de cette variable indépendante. Nous pourrons, dès Inrs, écrire :

$$
\begin{aligned}
& x=x_{0}+\mathrm{X}\left(v, y_{0}, \mathrm{~A}\right) \\
& y=y_{0}+\mathrm{Y}\left(v, y_{0}, \mathrm{~A}\right) \\
& \mathrm{X}\left(-v, y_{0}, \mathrm{~A}\right)=-\mathrm{X}\left(v, y_{0}, \mathrm{~A}\right) \\
& \mathrm{Y}\left(-v, y_{0}, \mathrm{~A}\right)=\mathrm{Y}\left(v, y_{0}, \mathrm{~A}\right) \\
& \mathrm{X}\left(v+2 \pi, y_{0}, \mathrm{~A}\right)=\mathrm{X}\left(v, y_{0}, \mathrm{~A}\right) \\
& \mathrm{Y}\left(v+2 \pi, y_{0}, \mathrm{~A}\right)=\mathrm{Y}\left(v, y_{0}, \mathrm{~A}\right)
\end{aligned}
$$

Il importe de se rappeler que $\lambda$ est une fonction, inconnue à priori, de $\mathrm{H}$, T et $\mathrm{A}$. D'après (3), on a donc :

$$
v=v\left(\mathrm{H}, \mathrm{T}, \mathrm{A}, x_{0}, t\right),
$$

en sorte que les fonctions $\mathrm{X}$ et $\mathrm{Y}$, telles qu'elles sont notées dans (5), dépendent de $\mathrm{A}$ à la fois directemeent et par l'intermédiaire de $v$.

$\S 10$. - Pour aller plus loin, il faut définir avec précision les propriétés de régularité de $\mathrm{X}$ et de Y, figurant dans (5).

Mme Dubrerl démontre que les composantes du vecleur vitesse admettent des dérivées partielles, continues en $x$ (donc en $t$ ) et en $y$ jusqu'au deuxième ordre. Nous admettrons que ce résultat entraîne le corollaire suivant : $\mathrm{X}$ et $\mathrm{Y}$ sont dérivables deux fois en $v$ et en $y_{0}$, les dérivées du second ordre étant suffisamment régulières pour rendre légitimes toutes les opérations que nous aurons à effectuer sur ces expressions. Il est, par ailleurs, asse $z$ facile, de déduire de [1] que tiques de $\mathrm{A}$, holomorphes, dans le voisinage de l'origine.

$\S 11$. - Nous sommes maintenant en mesure d'amorcer notre processus de calcul approché de la solution de notre problème. Les propriétés de régularité, énoncées au précédent paragraphe, montrent que les fonctions $\mathrm{X}, \mathrm{Y}$, périodiques et symétriques en $v$ (cf. (5)), sont développables dans D (cf. (4)), en série de Fourier, relativement à $v$, de la forme :

$x=x_{0}+\sum_{n=1}^{\infty} b_{n}\left(y_{0}, \mathrm{~A}\right) \sin n v$,
$y=y_{0}+\mathrm{F}\left(y_{0}, \mathrm{~A}\right)+\sum_{n=1}^{\infty} c_{n}\left(y_{0}, \mathrm{~A}\right) \cos n v$

absolument et uniformément convergentes dans $\mathrm{D}$, dérivables deux fois terme à terme dans ce domaine en $y_{o}$ et $v$ et dont les coefficients $\mathrm{F}$, $b_{n}$ et $c_{n}$ sont des fonctions holomorphes de l'amplitude A pour de petites valeurs de ce paramètre, nulles pour $\mathrm{A}=0$.

Comme nous cherchons une solution approchée, nous nous bornerons à calculer les développements limités des inconnues en A, arrêtés aux termes en $A^{3}$.

La suite des calculs montrera que $\left|b_{n}\right|$ et $\left|c_{n}\right|$ sont de l'ordre $\mathrm{A}^{n}$, pour $n \leqslant 3$. Nous espérons revenir ailleurs sur l'extension de ce résultat à $n$ quelconque. En attendant, rien ne permet d'affirmer à priori que nous n'avons pas laissé échapper un terme de premier ordre, par exemple, provenant de $b_{4}\left(y_{o}, \mathrm{~A}\right)$.

Pour simplifier les écritures, nous ferons usage des notations suivantes : les paramètres $\mathrm{H}$ et ' $\mathrm{T}$ seront supposés fixés; il est inutile, dès lors, de les expliciter comme arguments des éléments à calculer. Nous poserons alors :

$$
\left.\begin{array}{l}
v_{0}=\mu_{0} x_{0}-\nu t \\
\mu .(\mathrm{A})=\mu_{0}+\mathrm{A} \mu_{0}^{\prime}+\left(\mathrm{A}^{2} / 2\right) \mu_{0}{ }^{\prime \prime} \\
\mu_{0}=\mu(0) ; \\
\mu_{0}^{\prime}=(\partial \mu / \partial \mathrm{A})_{A=0} \\
\mu_{0}^{\prime \prime}=\left(\partial 2_{\mu} / \partial \mathrm{A}^{2}\right)_{A=0}
\end{array}\right\}
$$

On a, d'autre part :

$$
\left\{\begin{array}{l}
b_{n}=\mathrm{A} b_{n 1}+\left(\mathrm{A}^{2} / 2\right) b_{n 2}+\left(\mathrm{A}^{3} / 6\right) b_{n 3} ; n=1,2,3 \\
c_{n \cdot}=\mathrm{A} c_{n 1}+\left(\mathrm{A}^{2 / 2}\right) c_{n 2}+\left(\mathrm{A}^{3} / 6\right) c_{n 33} \\
\mathrm{~F}\left(y_{0}, \mathrm{~A}\right)=\mathrm{A} d_{1}+\left(\mathrm{A}^{2} / 2\right) \quad d_{2}+\left(\mathrm{A}^{3} / 6\right) d_{3}
\end{array}\right.
$$

formules où on a posé :

$$
\left\{\begin{array}{l}
b_{n p}\left(y_{0}\right)=\left.\left(\partial^{p} b_{n} / \partial \mathrm{A}^{p}\right)\right|_{\mathrm{A}=0} ; \\
c_{n p}\left(y_{0}\right)=\left.\left(\partial^{p} c_{n} / \partial \mathrm{A}^{p}\right)\right|_{\mathrm{A}=0} ; n, p=1,2,3 \\
d_{p}\left(y_{0}\right)=\left.\left(\partial^{p} \mathrm{~F} / \partial \mathrm{A}^{p}\right)\right|_{A=0} .
\end{array}\right.
$$

On vérifie aisément les formules approchées suivantes valables à $\mathrm{A}^{3}$ près :

$$
\left\{\begin{array}{l}
\sin n v=\sin n v_{0}+\mathrm{A} n x_{0} \mu_{0}^{\prime} \cos n v_{0}+\left(\mathrm{A}^{2} / 2\right)\left[n x_{0} \mu_{0}^{\prime \prime} \cos n v_{0}-n^{2} x_{0}{ }^{2} u_{0}{ }^{2} \sin n v_{0}\right] \\
\cos n v=\cos n v_{0}-\mathrm{A} n x_{0} \mu_{0}^{\prime} \sin n v_{0}-\left(\mathrm{A}^{2} / 2\right)\left[n x_{0} \mu_{0}^{\prime \prime} \sin n v_{0}+n^{2} x_{0}{ }^{2} u_{0}^{\prime}{ }^{2} \cos n v_{0}\right] .
\end{array}\right.
$$


En combinant (9) et (10), on trouve les développements limités, valables à $\mathrm{A}^{4}$ près :

$b_{n} \sin n v=\mathrm{A} b_{n 1} \sin n v_{0}+\mathrm{A}^{2}\left[\mu_{0}^{\prime} n x_{0} b_{n 1} \cos n v_{0}+1 / 2 b_{n 2} \sin n v\right]$,

$$
\left.\begin{array}{rl} 
& \left.+\mathrm{A}^{3} / 2\left[n x_{0}\left(\mu_{0}^{\prime \prime} b_{n 1}+\mu_{0}^{\prime} b_{n 2}\right) \cos n v_{0}+\left(-n^{2} x_{0}^{2} \mu_{0}^{\prime} b_{n 1}+b_{n 3} / 3\right)\right) \sin n v_{0}\right] ; \\
c_{n} \cos n v & =\mathrm{A} c_{n 1} \cos n v_{0}+\mathrm{A}^{2}\left[-\mu_{0}^{\prime} n x_{0} c_{n 1} \sin n v_{0}+(1 / 2) c_{n 2} \cos n v_{0}\right], \\
& +\mathrm{A}^{3} / 2\left[-n x_{0}\left(\mu_{0}^{\prime \prime} c_{n 1}+\mu_{0}^{\prime} c_{n 2}\right) \sin n v_{0}+\left(-n^{2} x_{0}^{2} \mu_{0}^{\prime} c_{n 1}+\left(c_{n 3} / 3\right)\right) \cos n v_{0}\right]
\end{array}\right\}
$$

Moyennant (9), $\left(9^{\prime}\right)$ et (11), le système (7) peut s'écrire, à $A^{ \pm}$près :

$$
\begin{aligned}
& x=x_{0}+\sum_{n=1}^{3} \mathrm{~A}^{n} \varphi_{n}, \\
& y=y_{0}+\sum_{n=1}^{3} \mathrm{~A}^{n} \psi_{n} ;
\end{aligned}
$$

où on a posé :

$$
\begin{aligned}
& \varphi_{1}=\sum_{n=1}^{3} b_{n 1} \sin n v_{0} ; \quad \\
& \psi_{1}=d_{1}+\sum_{1}^{3} c_{n 1} \cos n v_{0} . \\
& \varphi_{2}=\sum_{1}^{3}\left[\left(b_{n 2} / 2\right) \sin n v_{0}+n x_{0} \mu_{0}^{\prime} b_{n 1} \cos n v_{0}\right] \\
& \psi_{2}=\left(d_{2} / 2\right)+\sum_{1}^{3}\left[\left(c_{n 2} / 2\right) \cos n v_{0}-n x_{0} \mu_{0}{ }^{\prime} c_{n 1} \sin n v_{0}\right] \text {. } \\
& \varphi_{3}=(1 / 2) \sum_{1}^{3}\left[n x_{0}\left(\mu_{0}^{\prime \prime} b_{n 1}+\mu_{0}^{\prime} b_{n: 2}\right) \cos n v_{0}+\left(-n^{2} x_{0}{ }^{2} \mu_{\mu_{0}}^{\prime 2} b_{n 1}+\left(b_{n: 3} / 3\right)\right) \sin n v_{0}\right] \\
& \psi_{3}=\left(d_{3} / 6\right)+(1 / 2) \sum_{1}^{3}\left[-n x_{0}\left(u_{0}^{\prime \prime \prime} c_{n 1}+u_{0}^{\prime} c_{n 2}\right) \sin n v_{0}+\left(-n^{2} x_{01}{ }^{2} \mu_{0}{ }^{\prime 2} c_{n 1}+\left(c_{n 3} / 3\right)\right) \cos n v_{0}\right] \text {. }
\end{aligned}
$$
de $y_{0}$ :

Finalement, le problème posé revient à déterminer les paramètres $\mu_{0}, \mu_{0}^{\prime}, \mu_{0}^{\prime \prime}{ }_{0}$ et 21 fonctions

$$
b_{n p}, \quad c_{n p}, \quad d_{n}, \quad n, p=1,2,3
$$

$\S 12$. - Rappelons maintenant les équations du problème, formées par Miche (cf. [5]). En chaque point du domaine D (cf. (4)) les seconds membres de (7) doivent vérifier le système d'équations aux dérivées partielles :

$\frac{1}{\rho} \frac{\partial p}{\partial x_{0}}+\frac{\partial^{2} x}{\partial t^{2}} \frac{\partial x}{\partial x_{0}}+\left(\frac{\partial^{2} y}{\partial t^{2}}+g\right) \frac{\partial y}{\partial x_{0}}=0$
$\frac{1}{\rho} \frac{\partial p}{\partial y_{0}}+\frac{\partial^{2} x}{\partial t^{2}} \frac{\partial x}{\partial y_{0}}+\left(\frac{\partial^{2} y}{\partial t^{2}}+g\right) \frac{\partial y}{\partial y_{0}}=0$
$\frac{\mathrm{D}(x, y)}{\mathrm{D}\left(x_{0}, y_{0}\right)}=1$.

où $p$ est la pression et $\rho$ est la densité, constante, du liquide. Le système formé de $\left(16_{1}\right)$ et $\left(16_{2}\right)$ est l'analogue des équations classiques en variables de Lagrange; (16.3 $)$ est l'équation de continuité. On voit donc que (16) donne les équations générales du mouvement vertical d'un liquide pesant; dans ce paragraphe, il ne sera pas supposé que le régime à étudier soit du type houle.

En éliminant $p$ entre les deux premières relations (16), on a le système en $x$ et $y$ :

$$
\begin{aligned}
& \frac{\mathrm{D}\left(\partial^{2} x / \partial t^{2}, x\right)}{\mathrm{D}\left(x_{0}, y_{0}\right)}+\frac{\mathrm{D}\left(\partial^{2} y / d t^{2}, y\right)}{\mathrm{D}\left(x_{0}, !_{0}\right)}=0 ; \\
& \frac{\mathrm{D}(x, y)}{\mathrm{D}\left(x_{0}, y_{0}\right)}=1 .
\end{aligned}
$$

On sait d'ailleurs qu'à tout système de solutions $x, y$ de (17) correspond une solution $p\left(x_{0}, y_{0}, t\right)$ de (16), définie au moyen de deux quadratures, à une fonction additive, arbitraire, du temps près. Dans ce travail, nous avons uniquement en vue la détermination du vecteur déplacement; nous nous limiterons donc exclusivement à l'examen du système (17), laissant de côté le calcul, au reste laborieux, de $p$.

\$13. - En variables de Miche, les conditions 
aux limites sont faciles à écrire. Sur le fond : $y=-H$, les déplacements des particules doivent être horizontaux; d'où la première condition aux limites :

$$
\begin{aligned}
y-y_{0} & =0 \text { pour } y_{0}=-\mathrm{H}, \\
& -\infty \leqslant x_{0} \leqslant \infty, \quad-\infty \leqslant t<\infty
\end{aligned}
$$

Le long de la surface libre, la pression doit être constante. Il suffit, pour qu'il en soit ainsi, que :

$\partial p / \partial x_{0}=0$ pour $y_{0}=0,-\infty \leqslant x_{0} \leqslant \infty,-\infty \leqslant t \leqslant \infty$.

La relation précédente exprime, en effet, que le long de la ligne libre, image de $y_{0}=0$ par (7), la pression sera une fonction de $t$ seulement. Or $p$ n'est définie qu'à une fonction additive, arbitraire de cette variable. Il s'ensuit qu'on pourra toujours disposer de cette arbitraire pour rendre $p$ constant pour $y_{0}=0$, lorsque la relation précédente est satisfaite. D'après $\left(16_{2}\right)$, celle-ci est équivalente à :

$$
\begin{aligned}
& \frac{\partial^{2} x}{\partial t^{2}} \frac{\partial x}{\partial x_{0}}+\left(\frac{\partial^{2} y}{\partial t^{2}}+g\right) \frac{\partial y}{\partial x_{0}}=0 ; \\
& \quad \text { pour } y_{0}=0,-\infty \leqslant x_{0} \leqslant \infty,-\infty<t<+\infty .
\end{aligned}
$$

Ainsi, la condition à la surface libre peut s'exprimer au moyen des déplacements seuls.

$\S 14$. - Supposons maintenant que le mouvement étudiè du liquide soit reprèsentable au moyen des équations approchées du type (12); c'est dire que nous ne particularisons pas encore la forme des $\varphi_{n}$ et $\psi_{n}$. Portons dans (17), (18) et (19) les valeurs (12) des inconnues $x$ et $y$. Les relations ainsi obtenues doivent être identiquement vérifiées quel que soit A. Un raisonnement élémentaire et classique prouve alors que les coefficients des différentes puissances de A doivent être nuls, identiquement, dans les équations en cause. On trouve ainsi les systèmes vérifiés par les solutions approchées des divers ordres. Les calculs étant élémentaires, et, d'ailleurs, bien connus depuis les travaux de Miche, nous n'en donnerons que les résultats. En chaque point de D, on doit avoir :

$$
\begin{aligned}
& \left.\begin{array}{c}
\frac{\partial^{2}}{\partial t^{2}}\left(\frac{\partial \varphi_{1}}{\partial y_{0}}-\frac{\partial \psi_{1}}{\partial x_{0}}\right)=0 ; \\
\frac{\partial \varphi_{1}}{\partial x_{0}}+\frac{\partial \psi_{1}}{\partial y_{0}}=0 ;
\end{array}\right\} \\
& \left.\frac{\partial^{2}}{\partial t^{2}}\left(\frac{\partial \varphi_{2}}{\partial y_{0}}-\frac{\partial \psi_{2}}{\partial x_{0}}\right)=-\frac{\mathrm{D}\left\lceil\varphi_{1},\left(\partial^{2} \varphi_{1} / \partial t^{2}\right)\right]}{\mathrm{D}\left(x_{0}, y_{0}\right)}-\frac{\mathrm{D}\left[\psi_{1},\left(\partial^{2} \psi_{1} / \partial t^{2}\right)\right\rceil}{\mathrm{D}\left(x_{0}, y_{0}\right)}\right) \\
& \frac{\partial \varphi_{2}}{\partial x_{0}}+\frac{\partial \psi_{2}}{\partial y_{0}}=-\frac{\mathrm{D}\left(\varphi_{1}, \psi_{1}\right)}{\mathrm{D}\left(x_{0}, y_{0}\right)} \\
& \left.\frac{\partial^{2}}{\partial t^{2}}\left(\frac{\partial \varphi_{3}}{\partial y_{0}}-\frac{\partial \psi_{3}}{\partial x_{0}}\right)=-\left[\frac{\mathrm{D}\left[\varphi_{2},\left(\partial^{2} \varphi_{1} / \partial t^{2}\right)\right]}{\mathrm{D}\left(x_{0}, y_{0}\right)}+\frac{\mathrm{D}\left[\psi_{2},\left(\partial^{2} \psi_{1} / \partial t^{2}\right)\right]}{\mathrm{D}\left(x_{0}, y_{0}\right)}+\frac{\mathrm{D}\left[\varphi_{1},\left(\partial^{2} \varphi_{2} / d t^{2}\right)\right]}{\mathrm{D}\left(x_{0}, y_{0}\right)}+\frac{\mathrm{D}\left\lceil\psi_{1},\left(\partial^{2} \psi_{2} / \partial t^{2}\right)\right]}{\mathrm{D}\left(x_{0}, y_{0}\right)}\right]\right) \\
& \frac{\partial \varphi_{3}}{\partial x_{0}}+\frac{\partial \psi_{3}}{\partial y_{0}}=-\left[\frac{\mathrm{D}\left(\varphi_{1}, \psi_{2}\right)}{\mathrm{D}\left(x_{0}, y_{0}\right)}+\frac{\mathrm{D}\left(\varphi_{2}, \psi_{1}\right)}{\mathrm{D}\left(x_{0}, y_{0}\right)}\right]
\end{aligned}
$$

La condition aux limites (18) donne :

$$
\left.\psi_{n}\right|_{y=-\mathrm{H}} \equiv 0 \text {, pour }-\infty \leqslant x_{0} \leqslant \infty,-\infty \leqslant t \leqslant \infty, n=1,2,3
$$

Enfin, la pression sera constante au quatrième ordre près en A, le long de la surface libre, moyennant les trois relations valables quels que soient $x_{0}$ et $t$ :

$$
\begin{array}{ll}
\frac{\partial^{2} \varphi_{1}}{\partial t^{2}}+g \frac{\partial \psi_{1}}{\partial x_{0}}=0 ; & \text { pour } y_{0}=0 ; \\
\frac{\partial^{2} \varphi_{2}}{\partial t^{2}}+g \frac{\partial \psi_{2}}{\partial x_{0}}+\frac{\partial^{2} \varphi_{1}}{\partial t^{2}} \frac{\partial \varphi_{1}}{\partial x_{0}}+\frac{\partial^{2} \psi_{1}}{\partial t^{2}} \frac{\partial \psi_{1}}{\partial x_{0}}=0 ; & \text { pour } y_{0}=0 ; \\
\frac{\partial^{2} \varphi_{3}}{\partial t^{2}}+g \frac{\partial \psi_{3}}{\partial x_{0}}+\frac{\partial^{2} \varphi_{2}}{\partial t^{2}} \frac{\partial \varphi_{1}}{\partial x_{0}}+\frac{\partial^{2} \varphi_{1}}{\partial t^{2}} \frac{\partial \varphi_{2}}{\partial x_{0}}+\frac{\partial^{2} \psi_{2}}{\partial t^{2}} \frac{\partial \psi_{1}}{\partial x_{0}}+\frac{\partial^{2} \psi_{1}}{\partial t^{2}} \frac{\partial^{2} \psi_{2}}{\partial x_{0}}=0 ; \text { pour } y_{0}=0 ;
\end{array}
$$


Telles sont les équations du mouvement au troisième ordre près en A d'une couche horizontale, de profondeur constante, de liquide pesant, avec surface libre. Répétons que les seules hypothèses faites pour aboutir aux relations du précédent paragraphe consistent en ceci : les fonctions inconnues $x$ et $y$ admettent les développements limités du type (12), le domaine des variations des variables indépendantes $x_{0}, y_{0}$ étant la bande horizontale infinie $-\mathrm{H} \leqslant y_{0} \leqslant 0$. Ainsi donc, les formules précédentes valent pour une classe plus large de mouvements de liquides que les ondes périodiques de gravité.

Voyons les simplifications qui résultent pour les équations du problème lorsqu'on particularise au moyen des formules (13), (14) et (15) les approximations (12) des divers ordres.

Tout d'abord, les variables $x_{0}$ et $t$ n'interviennent que par l'intermédiaire de la variable auxiliaire $\nu$, définie par (8). Il s'ensuit que nous pourrions réduire d'une unité le nombre de variables indépendantes et utiliser pour les dérivations dans les formules de ce paragraphe les opérateurs :

$$
\frac{\partial}{\partial x_{0}}=\mu \frac{\partial}{\partial v} ; \quad \frac{\partial}{\partial t}=-v \frac{\partial}{\partial v}
$$

En second lieu, nous constaterons que chaque équation de ce paragraphe revient à écrire qu'une forme linéaire des $\sin n v_{0}$ et $\cos n v_{0}$, $n=1,2,3$, à coefficients dépendants de $y_{0}$ et de $x_{0}$ et des constantes inconnues, doit être identiquement nulle. Cela exige que tous les coefficients de la forme doivent être nuls identiquement; les relations ainsi obtenues suffisent à déterminer toutes les inconnues.

$\S 15$. - Avant de passer aux calculs, il est bon de marquer les différences du processus d'approximation que l'on vient de décrire avec celui de Miche. La variante du calcul utilisée par cet auteur ne donne pas le moyen d'évaluer, par un procédé régulier, les approximations des divers ordres de $\mu$ (A). D'autre part, Miche traite le cas de la houle quelconque. D'après ce qu'on a vu au $\S 5$, le vecteur déplacement n'est plus, en général, une fonction périodique du temps; en sorte qu'il n'est plus permis d'en écrire les composantes sous la forme simple (7). Dans le cas général, les seconds membres de ces relations contiennent nécessairement, et dès le premier ordre, des termes séculaires à coefficients arbitraires, dont le maniement est malaisé. Par contre, la méthode de Mrche conduit, comme on le verra, sans ambiguité, au but limité que nous nous sommes assignés; et, conformément à la théorie de Mme Dubreil, la suite des calculs n'introduit aucun élément arbitraire, à condition d'adopter les conclusions du paragraphe suivant.
$\S 16$. - Nous avons toujours appelé le paramètre A amplitude de l'onde. En fait, ce point est à préciser. D'après (12), on voit qu'une molécule déterminée s'écartera, en gros, d'autant plus de sa position moyenne $x_{0}, y_{0}$ que A sera plus grand. Il est donc naturel, dès lors, de considérer ce paramètre comme définissant l'amplitude du mouvement ondulatoire que nous cherchons à construire. Mais, cette définition n'est pas assez précise. En premier lieu, en effet, on peut remplacer A dans nos formules par tout autre paramètre positif $\mathrm{A}^{\prime}$, lié à $\mathrm{A}$ par la relation :

$$
\mathrm{A}=\mathrm{M}\left(\mathrm{A}^{\prime}\right) \text {, }
$$

où $\mathrm{M}$ est une fonction analytique, régulière pour $\mathrm{A}^{\prime}=0$, nulle pour $\mathrm{A}^{\prime}=0$, croissante de son argument, mais, à part cela, absolument quelconque. On peut donc inverser la relation précédente et écrire :

$$
A^{\prime}=M_{1}(A) \text {. }
$$

On a alors, au troisième ordre près en $\mathrm{A}^{\prime}$ :

$$
\mathrm{A}=a_{1} \mathrm{~A}^{\prime}+\left(a_{2} / 2\right) \mathrm{A}^{\prime 2}+\left(a_{3} / 6\right) \mathrm{A}^{\prime 3},
$$

les $a_{n}(n=1,2,3)$ étant des constantes à peu près arbitraires $\left(^{*}\right)$.

En portant dans $\left(12_{1}\right.$ ) la valeur de A ainsi définie, on aurait le développement limité au terme en $\mathrm{A}^{\prime 3}$ :

$$
\begin{aligned}
x=x_{0}+ & \mathrm{A}^{\prime} a_{1} \varphi_{1}+\mathrm{A}^{\prime 2}\left(a_{2} \varphi_{1}+a_{1}{ }^{2} \varphi_{2}\right) \\
& +\mathrm{A}^{\prime 3}\left(a_{3} \varphi_{1}+2 a_{1} a_{2} \varphi_{2}+a_{1}^{3} \varphi_{3}\right)
\end{aligned}
$$

On trouve donc une solution approchće du même type que $\left(12_{1}\right)$, mais avec une amplitude $A^{\prime}$ différente et un choix différent des approximations des divers ordres. On concoit qu'il soit loisible de tirer profit de cette indétermination pour simplifier les formules.

En second lieu, le mot amplitude a, en matière des mouvements ondulatoires, un sens physique précis : c'est la moitié de la dénivellation entre le creux et la crête de la surface libre d'une vague. Ainsi, la détermination précise de la grandeur ainsi définie exige la détermination préalable du profil de l'onde et ne peut, dès lors, être effectuée qu'une fois le problème résolu. Pour tourner cette difficulté, nous adopterons la convention suivante : le paramètre A devra être choisi de manière à être égal, à un facteur constant près, à l'amplitude du fondamental de la

(*) En fait, le second membre de (27) n'est astreint qu'à une seule condition : $a_{1}>0$ si on ne lui impose que la propriété d'être positif et croissant dans le voisinage de $A^{\prime}=0, A^{\prime}>0$. Bien entendu, d'autres inégalités sont a écrire si l'on veut que la propriété précédente soit vraie, pour un intervalle, fixé à priori, des variations de $A^{\prime}$. 
décomposition en série de Fourier de $y$ pour $y_{0}=0$. Une des raisons est que l'analyse harmonique d'un enregistrement de la surface libre donne aisément l'amplitude de ce fondamental. Les calculs seront bien simplifiés, en particulier les expressions de $x$ et $y$. Ceci revient à poser :

$$
\mathrm{A}=c_{1}(o, \mathrm{~A})
$$

Nous allons montrer qu'un changement de variable du type (27) permet toujours de se ramener au cas où :

$$
\begin{aligned}
& c_{11}(o)=1 ; \\
& c_{12}(o)=0 ; \\
& c_{13}(o)=0 .
\end{aligned}
$$

Affectons, en effet, d'un accent les quantités homologues aux quantités $c_{1 p}(o), p=1,2,3$ calculées avec le nouveau paramètre $\mathrm{A}^{\prime}$, lié à l'ancien par (27). On a :

$$
\begin{aligned}
& c_{11}^{\prime}(o)=a_{1} c_{11}(o) ; c_{12}{ }^{\prime}(o)=a_{1}^{2} c_{12}(o)+a_{2} c_{11}(o) ; \\
& c_{13}^{\prime}(o)=a_{1}^{3} c_{13}(o)+3 a_{1} a_{2} c_{12}(o)+a_{3} c_{11}(o) .
\end{aligned}
$$

Si donc $c_{11}(o) \neq 0$ - et on s'assurera ultérieurement qu'il en est bien ainsi - les relations précédentes permettent de calculer sans ambiguïté, de proche en proche, les quantités : $a_{1} \neq 0, a_{2}$ et $a_{3}$, telles que les $c^{\prime}{ }_{1 p}, p=1,2,3$ vérifient les conditions (30).

Dans toute la suite, les conditions (30) seront supposées remplies; elles complètent les conditions aux limites que nous avons décrites au $\S 14$.

Il nous reste maintenant à effectuer les calculs. Nous groupons la détermination des éléments de chaque ordre d'approximation dans un chapitre séparé. Les approximations des deux premiers ordres n'ajoutent rien de nouveau aux calculs, bien connus, de [5]; ce résultat est conforme à [1]. Par contre, le calcul des approximations d'ordre 3 nous paraît plus original.

\section{III. - SOLUTION DU PREMIER ORDRE}

$\S 17$. - Portons dans (20), les expressions (13) de $\varphi_{1}$ et $\psi_{1}$ : on trouve, en désignant par les accents les dérivations en $y_{0}$ :

$$
\begin{aligned}
d_{1}^{\prime}+ & \sum_{1}^{3}\left(\mu_{0} n b_{n 1} \cos n v_{0}+c_{n 1}^{\prime} \cos n v_{0}\right)=0 ; \\
& \sum_{1}^{3} n^{2}\left(b_{n 1}^{\prime}+n \mu_{0} c_{n 1}\right) \sin n v_{0}=0 .
\end{aligned}
$$

Les équations précédentes entraînent :

$$
\begin{aligned}
& d_{1}^{\prime}=0, \\
& \mu_{0} n b_{n 1}+c_{n 1}^{\prime}=0 ; \quad(n=1,2,3), \\
& b_{n 1}^{\prime}+\mu_{0} n c_{n 1}=0 .
\end{aligned}
$$

Le système différentiel ainsi obtenu s'intègre immédiatement. Compte tenu des conditions aux limites (23), on trouve :

$$
\left.\begin{array}{l}
d_{1}=0 ; \\
b_{n 1}=-k_{n 1} \operatorname{ch} n u_{0} ; \quad(n=1,2,3) \\
c_{n 1}=k_{n 1} \operatorname{sh} n u_{0},
\end{array}\right\}
$$

formules où les $k_{n 1}$ sont des constantes arbitraires et où on a posé :

$$
u_{0}=\mu_{0}\left(y_{0}+\mathrm{H}\right) \text {. }
$$

La condition (30) donne :

$$
\left.\begin{array}{c}
k_{11}=(1 / \operatorname{sh} q) ; \\
q=\mu_{0} \mathrm{H} .
\end{array}\right\}
$$

On aurait donc, d'après (13), (31) et (33) :

$$
\begin{aligned}
& \varphi_{1}=-\sum_{1}^{3} k_{\mathrm{n} 1} \operatorname{ch} n u_{0} \sin n v_{0} ; \\
& \psi_{1}=\sum_{1}^{3} k_{n 1} \operatorname{sh} n u_{0} \cos n v_{0} .
\end{aligned}
$$

$\S$ 18. - Ces expressions, portées dans (24), fournissent les conditions [cf. (32) et (33)] :

$$
\sum_{1}^{3} n k_{n 1}\left(n v^{2} \operatorname{ch} n q-\mu_{0} g \operatorname{sh} n q\right) \sin n v_{0}=0 .
$$

Si donc les $k_{\mathrm{n} 1}$ ne sont pas nuls, on doit avoir :

$$
v^{2}=\mu_{0} g \text { th } \mu_{0} \mathrm{H} \text {. }
$$

relation nécessaire, puisque, d'après (32), $k_{n} \neq 0$ et, éventuellement :

$$
n v^{2}=\mu_{0} g \text { th } n \mu_{0} \mathrm{H} .
$$

La condition (35) n'est autre que la célèbre formule d'Airy [cf. (8)]; dans tout ce awi suit. nous supposerons que $\mu_{0}$ est la racine unique de cette équation transcendante, où $v$ est une donnée. Voyons maintenant si, $\mu_{0}$ (ou $q$ ) ayant été choisi comme il vient d'être dit, les équations $\left(35^{\prime}\right)$ sont compatibles avec (35). D'après (33), on a, en divisant membre à membre $\left(35^{\prime}\right)$ et (35) :

$$
\frac{\text { th } n q}{n q}=\frac{\text { th } q}{q}
$$


où $n=2,3$, plus généralement où $n$ est un entier $>1$. Or, on vérifie sans peine que th $q / q$ est une fonction décroissante de $q$ pour $q>0$; il s'ensuit que la relation précédente est impossible. Aussi, $k_{21}$ et $k_{31}$ sont nuls et (34) peut s'écrire simplement :

$$
\begin{array}{ll}
\psi_{1}=k_{11} \operatorname{sh} u_{0} \cos v_{0} ; & b_{21}=b_{31}=0 ; \\
\varphi_{1}=-k_{11} \operatorname{ch} \varphi_{0} \sin v_{0} ; & c_{21}=c_{31}=0 .
\end{array}
$$

On retrouve les formules, équivalentes au premier ordre près, à celles, bien connues, de la houle linéaire de Stokes.

\section{IV. - SOLUTION DU SECOND ORDRE}

$\S 19$. - Nous allons reprendre la méthode suivie au $\S 17$. Portons dans les équations (21) les valeurs (14) de $\varphi_{2}$ et $\psi_{2}$ et les valeurs (36) de $\varphi_{1}$ et $\psi_{1}$. Après quelques calculs longs mais élémentaires, il vient :

$$
\begin{gathered}
\sum_{1}^{3} n^{2}\left(b_{n 2}^{\prime}+n \mu_{0} c_{n 2}\right) \sin n v_{0}+2 \mu_{0}^{\prime} c_{11} \sin v_{0}+2 \mu_{0}^{\prime} x_{0}\left(b_{11}^{\prime}+\mu_{0} c_{11}\right) \cos v_{0}=0 \\
2 d_{2}^{\prime}+\sum_{1}^{3}\left(n \mu_{0} b_{n 2}+c_{n 2}^{\prime}\right) \cos n v_{0}+2 \mu_{0}^{\prime} b_{11} \cos v_{0} \\
\quad-2 \mu_{0}^{\prime} x_{0}\left(\mu_{0} b_{11}+c_{11}^{\prime}\right) \sin v_{0}=k_{11}{ }^{2} \mu_{0,0}^{2}\left(\operatorname{ch} 2 u_{0}+\cos 2 v_{0}\right) .
\end{gathered}
$$

On abrègera beaucoup les calculs en utilisant la remarque finale du $\S 14$. Comme $v_{0}$ n'intervient dans $\varphi_{1}$ et $\psi_{1}$ que par ses sinus et cosinus, on aura, par exemple :

$$
\frac{\partial^{2} \varphi_{1}}{\partial t^{2}}=-\nu^{2} \varphi_{1}
$$

en sorte que $[\mathbf{c f}(21)]$ :

$$
\frac{\mathrm{D}\left[\varphi_{1},\left(\partial^{2} \varphi_{1} / \partial t^{2}\right)\right]}{\mathrm{D}\left(x_{0}, y_{0}\right)}=-\nu^{2} \frac{\mathrm{D}\left(\varphi_{1}, \psi_{1}\right)}{\mathrm{D}\left(x_{0}, y_{0}\right)}=0
$$

En appliquant la méthode du $\S 17$, le système précédent se décompose en annulant les coefficients des $\cos n v_{0}$ et $\sin n v_{0}, n=1,2,3$, dans chacune des équations du système, on trouve :

$$
\begin{aligned}
& 2 d_{2}^{\prime}=k_{11}^{2} \mu_{0}^{2} \operatorname{ch} 2 u_{0} ; \\
& \left\{\begin{array}{l}
3 u_{0} b_{32}+c_{32}^{\prime}=0 ; \\
b_{32}^{\prime}+3 \mu_{0} c_{32}=0 ;
\end{array}\right. \\
& \left\{\begin{array}{c}
\mu_{0} b_{12}+c_{12}^{\prime}=2 k_{11} \mu_{0}^{\prime} \operatorname{ch} u_{0} ; \\
b_{12}^{\prime}+\mu_{0} c_{12}=-2 k_{11} \mu_{0}^{\prime} \operatorname{sh} u_{0} .
\end{array}\right. \\
& \left\{\begin{array}{c}
2 \mu_{0} b_{22}+c_{22}^{\prime}=\mu_{0}^{2} k_{11^{2}}^{\prime 2} \\
b_{22}^{\prime}+2 \mu_{00} c_{22}=0
\end{array}\right.
\end{aligned}
$$

Le système précédent s'intègre encore sans peine. En tenant compte de (23), nous avons :

$$
\begin{array}{ll}
d_{2}=u_{0}\left(k_{11}{ }^{2} / 4\right) \operatorname{sh} 2 u_{0} ; & \left\{\begin{array}{l}
b_{22}=-k_{22} \operatorname{ch} 2 u_{0}+u_{0} \cdot k^{2}{ }_{11} / 2 ; \\
c_{22}=k_{22} \operatorname{sh} 2 u_{0} ;
\end{array}\right. \\
\left\{\begin{array}{l}
b_{32}=-k_{32} \operatorname{ch} 3 u_{0} ; \\
c_{32}=k_{32} \operatorname{sh} 3 u_{0} ;
\end{array}\right. & \left\{\begin{array}{l}
b_{12}=-k_{12} \operatorname{ch} u_{0}-2 k_{11}\left(\mu_{0}^{\prime} / \mu_{0}\right) u_{0} \operatorname{sh} u_{0} ; \\
c_{12}=k_{12} \operatorname{sh} u_{0}+2 k_{11}\left(\mu_{0}^{\prime} / \mu_{0}\right) u_{0} \operatorname{ch~} u_{0} ;
\end{array}\right.
\end{array}
$$

formules où les $k_{n 2}$ sont des constantes, pour le moment, arbitraires.

En vertu de (23), nous avons [cf. (32)] :

$$
k_{12}=-2 k_{11} \mu_{0}^{\prime} \mathrm{H} \operatorname{coth} q
$$

$\S 20$. - Enfin, la condition à la surface libre (25) va nous donner $k_{32}$, $k_{22}$ et $u_{0}^{\prime}$. Pour expliciter 
commodément cette relation, nous appliquerons encore les remarques finales du $\S 14$, en observant que [cf. (25) et (36)] :

$$
\frac{\partial^{2} \varphi_{1}}{\partial t^{2}} \frac{\partial \varphi_{1}}{\partial x_{0}}+\frac{\partial^{2} \psi_{1}}{\partial t^{2}} \frac{\partial \psi_{1}}{\partial x_{0}}=-\frac{\nu^{2}}{2} \frac{\partial}{\partial x_{0}}\left(\varphi_{1}^{2}+\psi_{1}^{2}\right)=-\frac{\nu^{2} k_{11}^{2}}{2} \frac{\partial}{\partial x_{0}}\left(\sin ^{2} v_{0}+\operatorname{sh}^{2} u_{0}\right) .
$$

Moyennant cela, (25) se réduit, tous calculs faits, à :

$$
\begin{aligned}
\sum_{1}^{3} \frac{n}{2}\left(\nu^{2} n b_{n 2}\right. & \left.+\mu_{0} g c_{n 2}\right) \sin n v_{0}+\frac{1}{2} \nu^{2} \mu_{0} k_{11^{2}} \sin 2 v_{0} \\
& +\mu_{0}^{\prime} x_{0}\left(\nu^{2} b_{11}+\mu_{0} g c_{11}\right) \cos v_{0}+g \mu_{0}^{\prime} c_{11} \sin v_{0}=0, \quad \text { pour } y_{0}=0 .
\end{aligned}
$$

Mais, d'après (31), (33) et (35) :

$$
v^{2} b_{11}+\mu_{0} g c_{11}=0, \quad \text { pour } y_{0}=0 .
$$

La condition à la surface libre sera done satisfaite si :

$$
\begin{aligned}
& 3 \nu^{2} b_{32}(o)+g \mu_{0} c_{32}(o)=0 ; \\
& 2 \nu^{2} b_{22}(o)+g \mu_{0} c_{22}(o)+1 / 2 \nu^{2} u_{0}^{2} k_{11}^{2}=0 . \\
& v^{2} b_{12}(o)+g u_{0} c_{12}(o)+2 u_{0}^{\prime} g c_{11}(o)=0 .
\end{aligned}
$$
s'écrit :

Remplacons dans ces relations les $b$ et les $c$ par leurs valeurs (30) (31) et (37). La première

$$
k_{32}\left(3 v^{2}-u_{0} g \text { th } 3 q\right)=0
$$

D'après ce qu'on a vu en discutant $\left(35^{\prime}\right)$, ceci ne peut être vérifié que si :

$$
k_{32}=0 .
$$

De $\left(39_{2}\right)$ on tire, compte tenu de (35):

$$
k_{22}(1-\operatorname{ch} 2 q)=-\frac{3}{2} \mu_{0} k_{11}^{2},
$$

d'où :

Enfin, $\left(39_{3}\right)$ donne :

$$
k_{22}=\frac{3}{4} \frac{\mu_{0}}{\operatorname{sh}^{4} q}=\frac{3}{4} k_{11}^{4} u_{0}
$$

$$
\frac{\mu_{0}^{\prime}}{\mu_{0}}\left(\frac{q}{\operatorname{sh} q \operatorname{ch} q}+1\right)=0
$$

ce qui entraîne, eu égard à (38) :

$$
\left\{\begin{array}{l}
\mu_{0}^{\prime}=0 \\
k_{12}=0
\end{array}\right.
$$
tableau :

$\S 21$. - En réunissant l'ensemble des résultats des deux précédents paragraphes, on trouve le

$$
\begin{aligned}
d_{2} & =u_{0} \frac{\operatorname{sh} 2 u_{0}}{4 \operatorname{sh}^{2} q} ; c_{32}=b_{32}=0 ; c_{12}=b_{12}=0 ; \\
b_{22} & =-\frac{3}{4} \frac{\mu_{0} \operatorname{ch}_{2} u_{0}}{\operatorname{sh}^{4} q}+\frac{\mu_{0}}{2 \operatorname{sh}^{2} q} ; \mu_{0}^{\prime}=0 ; \\
c_{22} & =\frac{3}{4} \frac{\mu_{01} \operatorname{sh}^{2} u_{0}}{\operatorname{sh}^{4} q}
\end{aligned}
$$

Portons ces résultats dans (14); il vient :

$$
\left.\begin{array}{l}
\varphi_{2}=\frac{\mu_{0}}{4 \operatorname{sh}^{2} q}\left[1-\frac{3}{2} \frac{\operatorname{ch} 2 u_{0}}{\operatorname{sh}^{2} q}\right] \sin 2 v_{0}=\frac{k^{2}{ }_{11} \mu_{0}}{4}\left[1-\frac{3}{2} k_{11}{ }^{2} \operatorname{ch} 2 u_{0}\right] \sin 2 v_{0} ; \\
\psi_{2}=\frac{u_{0}}{4 \operatorname{sh}^{2} q}\left[1+\frac{3}{2} \frac{\cos 2 v_{0}}{\operatorname{sh}^{2} q}\right] \operatorname{sh} 2 u_{0}=\frac{k^{2}{ }_{11} \mu_{0}}{4}\left[1+\frac{3}{2} k_{11}{ }^{2} \cos 2 v_{0}\right] \operatorname{sh} 2 u_{0}
\end{array}\right\}
$$


Les approximations du second ordre ainsi calculées sont identiques à celles de M. Miche. Mais cet auteur s'étant proposé un objectif moins limité, n'a pas pu obtenir la forme générale de l'approximation du second ordre. Au contraire, dans notre cas, nous sommes assurés de l'unicité de notre solution.

\section{V. - SOLUTION DU TROISIEME ORDRE}

$\S 22$. - Nous allons à présent expliciter les formules (22). Pour abréger les calculs, il est encore conmode d'utiliser les remarques finales du $\$ 14$. Observons que d'après (34) et (41):

$$
\begin{array}{lll}
\frac{\partial^{2} \varphi_{1}}{\partial t^{2}}=-v^{2} \varphi_{1} ; & \frac{\partial^{2} \varphi_{2}}{\partial t^{2}}=-4 \nu^{2} \varphi^{2} ; \\
\frac{\partial^{2} \psi_{1}}{\partial t^{2}}=-v^{2} \psi_{1} ; & \frac{\partial^{2} \psi_{2}}{\partial t^{2}}=-4 \nu^{2}\left(\psi_{2}-d_{2}\right) .
\end{array}
$$

Posons :

$$
\mathrm{Y}_{2}=\psi_{2}-d_{2}
$$

On voit alors que [cf. $\left.\left(22_{1}\right)\right]$ :

$$
\begin{aligned}
\frac{\mathrm{D}\left\lceil\left(\partial^{2} \varphi_{1} / \partial t^{2}\right), \varphi_{2}\right\rceil}{\mathrm{D}\left(x_{0}, y_{0}\right)}+\frac{\mathrm{D}\left\lceil\left(\partial^{2} \varphi_{2} / \partial t^{2}\right), \varphi_{1}\right]}{\mathrm{D}\left(x_{0}, y_{0}\right)}+\frac{\left.\mathrm{D}\left[\partial^{2} \psi_{1} / \partial t^{2}\right), \psi_{2}\right]}{\mathrm{D}\left(x_{0}, y_{0}\right)}+\frac{\left[\mathrm{D}\left(\partial^{2} \psi_{2} / \partial t^{2}\right), \psi_{1}\right]}{\mathrm{D}\left(x_{0}, y_{0}\right)} \\
=3 v^{2} \frac{\mathrm{D}\left(\varphi_{1}, \varphi_{2}\right)}{\mathrm{D}\left(x_{0}, y_{0}\right)}+3 v^{2} \frac{\mathrm{D}\left(\psi_{1}, Y_{2}\right)}{\mathrm{D}\left(x_{0}, y_{0}\right)}-v^{2} \frac{\mathrm{D})\left(\psi_{1}, d_{2}\right)}{\mathrm{D}\left(\boldsymbol{x}_{0}, y_{0}\right)}
\end{aligned}
$$

Pour faciliter la tâche du lecteur, nous explicitons les valeurs des expressions ainsi introduites. On trouve successivement:

$$
\begin{aligned}
3 \nu^{2} \frac{\mathrm{D}\left(\varphi_{1}, \varphi_{2}\right)}{\mathrm{D}\left(x_{0}, y_{0}\right)} & =\frac{3}{8} \nu^{2} k_{11^{3} \mu_{0}{ }^{3}}\left[\left(-2 \operatorname{sh} u_{0}+\frac{3}{\operatorname{sh}^{2} q} \operatorname{sh} 3 u_{0}\right) \sin v_{0}+\left(2+\frac{3}{\operatorname{sh}^{2} q}\right) \operatorname{sh} u_{0} \sin 3 v_{0} ;\right] \\
3 \nu^{2} \frac{\mathrm{D}\left(\psi_{1}, \mathrm{Y}_{2}\right)}{\mathrm{D}\left(x_{0}, y_{0}\right)} & =\frac{9}{8} \frac{\nu^{2} k_{11} \mu_{0}^{3}}{\operatorname{sh}^{2} q}\left[\operatorname{sh} 3 u_{0} \sin v_{0}+\operatorname{sh} u_{0} \sin 3 v_{0}\right] \\
-\nu^{2} \frac{\mathrm{D}\left(\psi_{1}, d_{2}\right)}{\mathrm{D}\left(x_{0}, y_{0}\right)} & =\frac{\nu^{2} k_{11}{ }^{3} u_{0}{ }^{3}}{4}\left[-\operatorname{sh} u_{0}+\operatorname{sh} 3 u_{0}\right] \sin v_{0}
\end{aligned}
$$

A voir la forme des seconds membres, on comprend qu'il y a intérêt à ne pas remplacer $k_{11}$ par sa valeur (33). La somme des trois expressions précédentes s'écrit:

$$
\frac{v^{2} k_{11}{ }^{3} u_{0}^{3}}{4}\left\{\left[\left(\frac{9}{\operatorname{sh}^{2} q}+1\right) \operatorname{sh} 3 u_{0}-4 \operatorname{sh} u_{0}\right] \sin v_{0}+3\left(\frac{3}{\operatorname{sh}^{2} q}+1\right) \operatorname{sh} u_{0} \sin 3 v_{0}\right\}
$$

Enfin, à partir de (15), (36) et (40), il est facile de vérifier la relation :

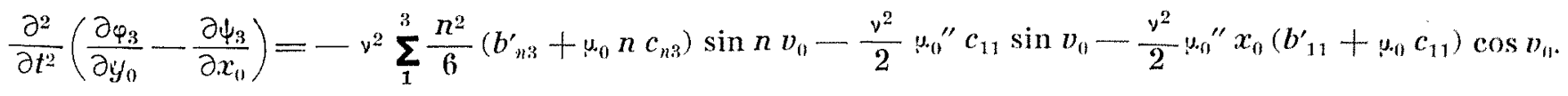

En égalant les deux dernières expressions ou formes $\left(25_{1}\right)$, en annulant les coefficients des $\cos n v_{0}$ et $\sin n v_{0}$, on trouve les relations :

$$
\begin{aligned}
\frac{1}{6}\left(b_{13}^{\prime}+\mu_{0} c_{13}\right)+\frac{1}{2} k_{11} \mu_{0}^{\prime \prime} \operatorname{sh} u_{0} & =-\frac{k_{11}^{3} \mu_{00}^{3}}{4}\left[\frac{q}{\operatorname{sh}^{2} q}+1\right] \operatorname{sh} 3 u_{0}+k_{11^{3} u_{00}^{3}{ }^{3} \operatorname{sh} u_{0}} \\
b_{23}+2 \mu_{0} c_{23}=0 & \\
\frac{3}{2}\left(b_{33}^{\prime}+3 \mu_{0} c_{33}\right) & =-\frac{3}{4} k_{11}^{3} u_{01}^{3}\left(\frac{3}{\operatorname{sh}^{2} q}+1\right) \operatorname{sh} u_{01}
\end{aligned}
$$


Moyennant (42), l'équation de continuité au troisième ordre $\left(25_{2}\right)$ peut être formée sans peine. Il vient, eu égard à (15), (36) et (40) :

$$
\begin{gathered}
d_{3}^{\prime}+\frac{1}{6} \sum_{1}\left(n u_{0} b_{n 3}+c_{n 3}^{\prime}\right) \cos n v_{0}+\frac{1}{2} \mu_{0}^{\prime \prime} b_{11} \cos v_{0}-\frac{1}{2} \mu_{0}^{\prime \prime} x_{0}\left(\mu_{0} b_{11}+c^{\prime}{ }_{11}\right) \sin v_{0}= \\
=\frac{k_{11}^{3} \mu_{0}^{3}}{4}\left[\left(\frac{3}{\operatorname{sh}^{2} q}+1\right) \operatorname{ch~} 3 u_{0} \cos v_{0}+\left(\frac{3}{\operatorname{sh}^{2} q}-1\right) \operatorname{ch~} u_{0} \cos 3 v_{0} \mid\right.
\end{gathered}
$$

On en déduit, en observant que, d'après les résultats du $\S 17$,

$$
\begin{aligned}
& u_{0} b_{11}+c^{\prime}{ }_{11}=0 \text { : } \\
& t_{3}^{\prime}=0 \\
& \frac{1}{6}\left(\mu_{0} b_{13}+c^{\prime}{ }_{13}\right)+\frac{1}{2} \mu_{{ }_{0}}^{\prime \prime} b_{11}=\frac{k_{11^{3} \mu_{0}{ }^{3}}}{4}\left(1+\frac{3}{\operatorname{sh}^{2} q}\right) \operatorname{ch} 3 u_{0} ; \\
& 2 u_{0} b_{23}+c_{23}^{\prime}=0 \\
& \frac{1}{6}\left(3 u_{0} b_{33}+c_{33}^{\prime}\right)=\frac{k_{11}^{3} \mu_{0}^{3}}{4}\left(\frac{3}{\operatorname{sh}^{2} q}-1\right) \operatorname{ch} n_{0} .
\end{aligned}
$$

Telles sont les équations indéfinies du problème.

$\S 23$. - En combinant (42) et (44) et en tenant compte de (23), on voit d'abord que :

$$
\left.\begin{array}{l}
d_{3}=0 ; \\
b_{23}=-k_{23} \operatorname{ch} 2 u_{0} ; \\
c_{23}=\quad k_{23} \operatorname{sh} 2 u_{0},
\end{array}\right\}
$$

où $k_{23}$ est une constante, pour le moment arbitraire. Le système $\left(42_{1}\right)$ et $\left(44_{2}\right)$ définit les fonctions inconnues $b_{13}$ et $c_{13}$; en éliminant $b_{13}$ entre les deux équations du système, on trouve l'équation différentielle :

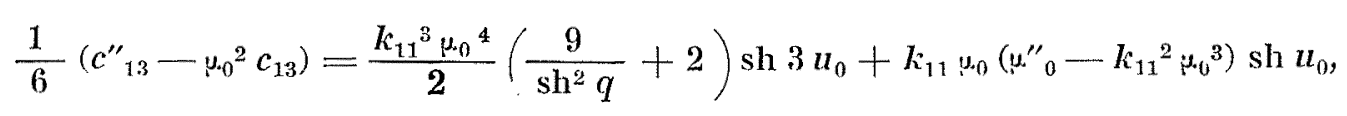

dont l'intégrale générale s'écrit :

$$
c_{13}=k_{13} \operatorname{sh}\left(u_{0}+c\right)+\alpha u_{0} \operatorname{ch} u_{0}+\beta \operatorname{sh} 3 u_{0},
$$

où $k_{13}$ et $c$ sont des constantes arbitraires et où on a posé:

$$
\left.\begin{array}{l}
\alpha=3 k_{11}\left(\frac{\mu_{0}^{\prime \prime}}{\mu_{0}}-k_{11}^{2} \mu_{0}^{2}\right) ; \\
\beta=\frac{3}{8} k_{11}^{3} \mu_{0}^{2}\left(\frac{9}{\operatorname{sh}^{2} q}+2\right)
\end{array}\right\}
$$

Observons que les deux derniers termes de l'expression de $c_{13}$ (qui représentent une solution particulière de l'équation de définition de cette inconnue) s'annulent pour $y_{0}=\mathrm{H}$, c'est-à-dire, d'après (31), pour $u_{0}=0$. La condition (23) sera donc satisfaite si $c=0$; finalement :

$$
c_{13}=k_{13} \operatorname{sh} u_{0}+\alpha u_{0} \operatorname{ch} u_{0}+\beta \operatorname{sh} 3 u_{0}
$$

L'équation $\left(44_{2}\right)$ définit alors $b_{13}$ sans aucune ambiguïté et on trouve :

$$
b_{13}=-k_{13} \operatorname{ch} u_{0}+\alpha_{1} \operatorname{ch} u_{0}-\alpha u_{0} \operatorname{sh} u_{0}+\beta_{1} \operatorname{ch} 3 u_{0},
$$

où $\alpha$ et $\beta$ sont donnéş par (45) et où on a posé :

$$
\left.\begin{array}{l}
\alpha_{1}=3 k_{11}^{3} \mu_{0}^{2} ; \\
\beta_{1}=-\frac{3}{4} k_{11}^{3} \mu_{0}^{2}\left[\frac{15}{\operatorname{sh}^{2} q}+1\right]
\end{array}\right\}
$$



et $\mu_{0}^{\prime \prime}$ :

Ecrivons, maintenant, que $c_{13}$ satisfait à (30); on a ainsi une relation entre les paramètres $k_{13}$

$$
k_{13}=3 q \frac{\operatorname{ch} q}{\operatorname{sh}^{2} q}\left(\frac{\mu_{0}^{2}}{\operatorname{sh}^{2} q}-\frac{\mu_{0}^{\prime \prime}{ }_{0}}{\mu_{0}}\right)-\frac{3}{8} \frac{\operatorname{sh}^{2} 3 q}{\operatorname{sh}^{4} q}\left(\frac{9}{\operatorname{sh}^{2} q}+2 .\right)
$$

Il reste à résoudre le système $\left(42_{3}\right)$ et $\left(44_{4}\right)$ en $b_{33}$ et $c_{33}$. Eliminons encore $b_{33}$ entre ces deux relations; on a :

$$
c^{\prime \prime}{ }_{33}-9 \mu_{0}^{2} c_{33}=9 k_{11^{3}} \mu_{0}{ }^{4} \frac{\operatorname{sh} u_{0}}{\operatorname{sh}^{2} q}
$$

L'équation avec second membre admet la solution particuliẹre $\gamma \operatorname{sh} u_{0}$, avec:

$$
\gamma=-\frac{9}{8} \frac{k_{11}^{3} \mu_{0}^{2}}{\operatorname{sh}^{2} q}
$$

en sorte que son intégrale générale s'écril :

$$
c_{33}=k_{33} \operatorname{sh} 3\left(u_{0}+c\right)+\gamma \operatorname{sh} u_{0},
$$

où $k_{333}$ et $c$ sont encore des constantes arbitraires. La condition (23) sera vérifiće si $c=0$ ct :

$$
c_{33}=k_{: 33} \operatorname{sh} 3 u_{0}+\gamma \operatorname{sh} u_{0} .
$$

Il résulte alors de $\left(44_{3}\right)$ que :

$$
b_{33}=-k_{33} \operatorname{ch} 3 u_{0}+\gamma_{1} \operatorname{ch} u_{0},
$$

où on a posé :

$$
\gamma_{1}=\frac{k_{11}^{3} u_{0}^{2}}{8}\left[\frac{15}{\operatorname{sh}^{2} q}-4\right]
$$

\$24. - Ecrivons maintenant la condition (26) à la surface libre. Utilisons les remarques et les notations du $\$ 22$. Nous avons :

$$
\begin{aligned}
Q & =\frac{\partial^{2} \varphi_{2}}{\partial t^{2}} \frac{\partial \varphi_{1}}{\partial x_{1}}+\frac{\partial^{2} \varphi_{1}}{\partial t^{2}} \frac{\partial \varphi_{1}}{\partial x_{0}}+\frac{\partial^{2} \psi_{2}}{\partial t^{2}} \frac{\partial \psi_{1}}{\partial x_{0}}+\frac{\partial^{2} \psi_{1}}{\partial t^{2}} \frac{\partial \psi_{2}}{\partial x_{0}} \\
& =-\left.v^{2}\right|^{-} 4\left(\varphi_{2} \frac{\partial \varphi_{1}}{\partial x_{0}}+Y_{2} \frac{\partial \psi_{1}}{\partial x_{0}}\right)+\varphi_{1} \frac{\partial \varphi_{2}}{\partial x_{0}}+\psi_{1} \frac{\partial Y_{2}}{\partial x_{0}} \mid \\
& \left.=-\left.v^{2}\right|^{2}\left(\varphi_{2} \frac{\partial \varphi_{1}}{\partial x_{0}}+Y_{2} \frac{\partial \psi_{1}}{\partial x_{0}}\right)+\frac{\partial}{\partial x_{0}}\left(\varphi_{1} \varphi_{2}+\psi_{1} Y_{2}\right)\right] \mid
\end{aligned}
$$

Or, de (36) et de (41), on tire, après quelques calculs faciles mais longs :

$$
\varphi_{1} \varphi_{2}+\psi_{1} \mathrm{Y}_{2}=\frac{k_{11}{ }^{3} u_{0}^{2}}{8}\left[\left(\frac{3}{2} k_{\mathrm{r}_{1}}^{2} \operatorname{ch} 3 u_{0}-\operatorname{ch} u_{0}\right) \cos v_{0}-\left(\frac{3}{2} k_{11}^{2} \operatorname{ch} u_{0}-\operatorname{ch} u_{0}\right) \cos 3 v_{0}\right]
$$

et :

$$
\varphi_{2} \frac{\partial \varphi_{1}}{d x_{0}}+Y_{2} \frac{\partial \psi_{1}}{\partial x_{0}}=\frac{k_{11} \mu_{0}^{2}}{8}\left[\left(\frac{3}{2} k_{11}^{2} \operatorname{ch} 3 u_{0}-\operatorname{ch} u_{0}\right) \sin v_{0}+\left(\frac{3}{2} k_{11}^{2} \operatorname{ch} u_{0}-\operatorname{ch} u_{0}\right) \sin 3 v_{0}\right]
$$

En portant ces expressions dans l'équation de définition de ci-dessus, il vient :

$$
\mathrm{Q}=\frac{\nu_{2} k_{11^{3} u_{0}{ }^{2}}}{8}\left[\left(2 \operatorname{ch} u_{0}-3 k_{11} \operatorname{ch} 3 u_{0}\right) \sin v_{0}+\left(6-9 k_{11^{2}}\right) \operatorname{ch} u_{0} \sin 3 v_{0}\right\rceil
$$

D'un autre côté, (15) donne, eu égard à (36) et à (40) :

$$
\begin{aligned}
\mathrm{R}=\frac{\partial^{2} \varphi_{3}}{\partial t^{2}}+g \frac{\partial \psi_{3}}{\partial x_{0}}=-\sum_{1}^{3} \frac{n}{6}\left(n \nu^{2} b_{n 3}+\mu_{0} g c_{n 3}\right) \sin n v_{0} \\
\quad-\frac{\mu_{n}^{\prime \prime} x_{0}}{2}\left(\nu^{2} b_{11}+\mu_{0} g c_{11}\right) \cos v_{0}-\frac{1}{2} g \mu_{00}^{\prime \prime} c_{11} \sin v_{0} .
\end{aligned}
$$


Ơr, d'après (54) et (57), (26) s'écrit :

$$
\mathrm{Q}+\mathrm{R}=0, \text { pour } y_{0}=0 \text {. }
$$

En portant dans cette relation les valeurs (56) et (57) des deux membres et en identifiant les coefficients des $\cos n v_{0}$ et des $\sin n v_{0}$, on trouve les conditions suivantes, valables pour $y_{0}=0$ :

$$
\begin{aligned}
& \nu^{2} b_{13}+u_{0} g c_{13}+3 g \mu_{0}^{\prime \prime} c_{11}=\frac{3}{4} \nu^{2} k_{11}{ }^{3} \mu_{0}{ }^{2}\left(2 \operatorname{ch} u_{0}-3 k_{11^{2}} \operatorname{ch} 3 u_{4}\right) ; \\
& 2 \nu^{2} b_{23}+\mu_{0} g c_{23}=0 \\
& 3 \nu^{2} b_{33}+u_{0} g c_{33}=\frac{3}{2} \nu^{2} k_{11}{ }^{3} \mu_{0}{ }^{2}\left(1-\frac{3}{2} k_{11^{2}}{ }^{2}\right) \operatorname{ch} u_{0} .
\end{aligned}
$$

D'après $\left(44^{\prime}\right)$ et $\left(58_{2}\right)$, on voit que $[\mathrm{cf} .(32)$ et $(33)]$ :

$$
k_{23}\left(2 \nu^{2} \operatorname{ch} 2 q-\mu_{0} g \operatorname{sh} 2 q\right)=0 .
$$

Or, la condition $\left(35^{\prime}\right)$ ne peut être vérifiée que pour $n=1$ (cf. $\S 18$ ); on conclut de là :

$$
k_{23}=0 \text {, }
$$

en sorte que, eu égard à $\left(44^{\prime}\right)$ :

$$
\left.\begin{array}{l}
b_{23} \equiv 0 ; \\
c_{23} \equiv 0 .
\end{array}\right\}
$$

Faisons maintenant $y_{0}=0$ dans $\left(58_{1}\right)$; remplaçons-y $: c_{11}, c_{13}$ et $b_{13}$ par leurs valeurs (31) et (33), (46) et (47). Si on tient compte de (32), le résultat s'écrit :

$k_{13}\left(\mu_{0} g \operatorname{sh} q-v^{2} \operatorname{ch} q\right)+v^{2} \alpha_{1} \operatorname{ch} q+\alpha q\left(\mu_{0} g \operatorname{ch} q-v^{2} \operatorname{sh} q\right)+v^{2} \beta_{1} \operatorname{ch} 3 q$

$$
+\mu_{0} g \beta \operatorname{sh} 3 q+3 g u^{\prime \prime}{ }_{0}=\frac{3}{4} v^{2} k_{11}{ }^{3} u_{0}^{2}\left(2 \operatorname{ch} q-3 k_{11}^{2} \operatorname{ch} 3 q\right) \text {. }
$$

D'après (35), le coefficient de $k_{13}$ du premier membre est nul. Remplaçons alors dans l'équation précédente $\nu^{2}$ par sa valeur (35), $\alpha, \alpha_{1}, \beta, \beta_{1}$ par leurs valeurs (45) et (48) respectivement; après quelques calculs un peu longs, nous aurons :

$$
\mu_{0}^{\prime \prime}=\frac{\mu_{0}{ }^{3}}{\operatorname{sh}^{2} q} \frac{2 q-\left\lceil\left(9 / 4 \operatorname{sh}^{2} q\right)+1\right\rceil \operatorname{sh} 2 q}{2 q+\operatorname{sh} 2 q}
$$
$y_{0}=0$ :

Il reste à discuter $\left(58_{3}\right)$. Compte tenu de (51) et de (52), cette équation s'écrit, si l'on y fait

$$
k_{33}\left(u_{0} g \operatorname{sh} 3 q-3 \nu^{2} \operatorname{ch} 3 q\right)+3 \nu^{2} \gamma_{1} \operatorname{ch} q+\mu_{0} g \gamma \operatorname{sh} q=\frac{3}{2} \nu^{2} k_{11}{ }^{3} \mu_{0}{ }^{2}\left(1-\frac{3}{2 \operatorname{sh}^{2} q}\right) \operatorname{ch} q
$$

Remplaçons alors dans la relation précédente $\gamma$ et $\gamma_{1}$ par leurs valeurs (50) et (53); en effectuant les calculs, on trouve :

$$
k_{33}=\frac{3}{4} \frac{\mu_{0}{ }^{2}}{\operatorname{sh}^{2} q} \frac{9 \operatorname{coth} q-2 \operatorname{sh} 2 q}{\operatorname{sh} 4 q-2 \operatorname{sh} 2 q}
$$

\$ 25. - Ces dernières formules achèvent la solution complète du problème que nous nous sommes posé. Pour la commodité du lecteur, nous allons réunir en un tableau les formules relatives à la solution du troisième ordre.

D'après $\left(44^{\prime}\right),(46),(47),(51)$ et $(52)$, nous avons :

$$
\begin{aligned}
& d_{3}=0 ; \\
& b_{13}=-k_{13} \operatorname{ch} u_{0}+\alpha_{1} \operatorname{ch} u_{0}-\alpha u_{0} \operatorname{sh} u_{0}+\beta_{1} \operatorname{ch} 3 u_{0} ; \\
& c_{13}=\quad k_{13} \operatorname{sh} u_{0}+\alpha u_{0} \operatorname{ch} u_{0}+\beta \operatorname{sh} 3 u_{0},
\end{aligned}
$$


formules où on a posé [cf. (32), (33), (45), (48) et (49)] :

$$
\begin{aligned}
& u_{0}=\mu_{0}\left(y_{0}+\mathrm{H}\right) ; \quad q=\mu_{0} \mathrm{H} ; \\
& \alpha=\frac{3}{\operatorname{sh} q}\left[\frac{\mu_{0}^{\prime \prime}}{\mu_{0}}-\frac{\mu_{0}{ }^{2}}{\operatorname{sh}^{2} q}\right] \quad ; \beta=\frac{3}{8} \frac{\mu_{0}^{2}}{\operatorname{sh}^{3} q}\left[\frac{9}{\operatorname{sh}^{2} q}+2\right] ; \\
& \alpha_{1}=3 \frac{\mu_{0}{ }^{2}}{\operatorname{sh}^{3} q} \quad ; \beta_{1}=-\frac{3}{4} \frac{\mu_{0}^{2}}{\operatorname{sh}^{2} q}\left[\frac{15}{\operatorname{sh}^{2} q}+1\right] \\
& k_{13}=3 q \frac{\operatorname{ch} q}{\operatorname{sh}^{2} q}\left(\frac{\mu_{0}^{2}}{\operatorname{sh}^{2} q}-\frac{\mu_{0}^{\prime \prime}}{\mu_{0}}\right)-\frac{3}{8} \frac{\operatorname{sh}^{3} q}{\operatorname{sh}^{4} q} \mu_{0}^{2}\left[\frac{9}{\operatorname{sh}^{2} q}+2\right] ;
\end{aligned}
$$

et où ب.." ${ }_{0}$ s'exprime en fonction des données au moỵen de $(60)$.

D'après (59), on a :

$$
\begin{aligned}
& b_{23} \equiv 0 \\
& c_{23} \equiv 0
\end{aligned}
$$

Enfin [cf. (51) et (52)], il vient :

$$
\begin{gathered}
b_{33}=-k_{33} \operatorname{ch} 3 u_{0}+\gamma_{1} \operatorname{ch} u_{0} ; \\
c_{33}=k_{33} \operatorname{sh} 3 u_{0}+\gamma \operatorname{sh} u_{0},
\end{gathered}
$$

où il faut poser, d'après (50), (53) et (61):

$$
\begin{aligned}
& r=-\frac{9}{8} \frac{\mu_{0}^{2}}{\operatorname{sh}^{5} q} ; \\
& \gamma_{1}=\frac{\mu_{0}{ }^{2}}{8 \operatorname{sh}^{3} q}\left[\frac{15}{\operatorname{sh}^{2} q}-4\right],
\end{aligned}
$$

$$
k_{33}=\frac{3}{4} \frac{\mu_{0}{ }^{2}}{\operatorname{sh}^{3} q} \frac{9 \operatorname{coth} q-2 \operatorname{sh} 2 q}{\operatorname{sh} 4 q-2 \operatorname{sh} 2 q}
$$

Ceci étant, les composantes du troisième ordre du vecteur-déplacement $\left(x-x_{0}\right)$ et $\left(y-y_{0}\right)$, à savoir $\mathrm{A}^{3} \varphi_{3}$ et $\mathrm{A}^{3} \psi_{3}$, seront, d'après (15), (36) et (40), donnés par :

$$
\begin{aligned}
& \varphi_{3}=\frac{1}{2}\left[\frac{1}{3}\left(b_{33} \sin 3 v_{0}+b_{13} \sin v_{0}\right)-u_{0}^{\prime \prime} x_{0} \frac{\operatorname{ch} u_{0}}{\operatorname{sh} q} \cos v_{0}\right] ; \\
& \varphi_{3}=\frac{1}{2}\left[\frac{1}{3}\left(c_{33} \cos 3 v_{0}+c_{13} \cos v_{0}\right)-u_{0}^{\prime \prime} x_{0} \frac{\operatorname{sh} u_{0}}{\operatorname{sh} q} \sin v_{0}\right] .
\end{aligned}
$$

$\S 26$. - Il nous reste maintenant à présenter au sujet de ces résultats quelques remarques. Ơn constate aisément que les formules résolutives (12), compte tenu de (8), (9), (9'), (10), (13), (14) et (15) peuvent être condensées sous la forme :

$$
\begin{aligned}
& x=x_{0}+\mathrm{B}_{13}\left(y_{0}, \mathrm{~A}\right) \sin v_{2}+\mathrm{B}_{22}\left(y_{0}, \mathrm{~A}\right) \sin 2 v_{1}+\mathrm{B}_{31}\left(y_{0}, \mathrm{~A}\right) \sin 3 v_{01} ; \\
& y=y_{0}+\mathrm{F}_{2}\left(y_{0}, \mathrm{~A}\right)+\mathrm{C}_{13}\left(y_{0}, \mathrm{~A}\right) \cos v_{2}+\mathrm{C}_{22}\left(y_{0}, \mathrm{~A}\right) \cos 2 v_{1}+\mathrm{C}_{31}\left(y_{0}, \mathrm{~A}\right) \cos 3 v_{0},
\end{aligned}
$$

où on a posé $[$ cf. (6)] :

$$
\begin{array}{cc}
v_{2}=v_{0}+\left.\mathrm{A} \frac{\partial v_{0}}{\partial \mathrm{A}}\right|_{\mathrm{A}=0}+\left.\frac{\mathrm{A}^{2}}{2} \frac{\partial^{2} p_{0}}{\partial \mathrm{A}^{2}}\right|_{\mathrm{A}=0} & \\
v_{1}=v_{0}+\left.\mathrm{A} \frac{\partial v_{0}}{\partial \mathrm{A}}\right|_{\Lambda=0} & n=1,2,3 \\
\mathrm{~B}_{n ; p}\left(y_{0}\right)=\left.\sum_{i=1}^{p} \frac{\mathrm{A}^{i}}{i !} \frac{\partial^{i} b_{n}}{\partial \mathrm{A}^{i}}\right|_{\mathrm{A}=0} ; & p=1,2,3
\end{array}
$$

la définition des $\mathrm{C}_{n p}$ étant analogue à celle des $\mathrm{B}_{n p}$. Ces remarques évidentes peuvent constituer le point de départ d'une étude des lois de récurrence dans la formation des inconnues. Nous espérons avoir une occasion de revenir sur ce point. 
$\S 27$. - Récrivons la formule [cf. (40)] :

$$
\mu=\mu_{0}+\left(\mathrm{A}^{2} / 2\right) \mu_{0}^{\prime \prime} .
$$

D'après (60), ceci s'explicite comme il suit :

$$
\mu=\mu_{0}+\frac{A^{2}}{2} \frac{\mu_{0}^{3}}{\operatorname{sh}^{2} \mu_{0} \mathrm{H}} \frac{2 \mu_{0} \mathrm{H}-\left[\left(9 / 4 \operatorname{sh}^{2} \mu_{0} \mathrm{H}\right)+1\right\rceil \operatorname{sh} 2 \mu_{0} \mathrm{H}}{2 \mu_{0} \mathrm{H}+\operatorname{sh} 2 \mu_{0} \mathrm{H}}
$$

En vertu de (3), on aurait donc, en négligeant les quantités d'ordre $\mathrm{A}^{3}$ au moins, l'expression ci-après de la longueur d'onde :

$$
\lambda=\lambda_{0}\left[1+\frac{\pi^{2}}{2 \operatorname{sh}^{2}\left(2 \pi \mathrm{H} / \lambda_{0}\right)} \frac{4 A^{2}}{\lambda_{0}{ }^{2}} \frac{\left\lceil 9 / 4 \mathrm{sh}^{2} 2 \pi\left(\mathrm{H} / \lambda_{0}\right)+1\right\rceil \operatorname{sh} 4 \pi\left(\mathrm{H} / \lambda_{0}\right)-4 \pi\left(\mathrm{H} / \mathrm{\lambda}_{0}\right)}{\operatorname{sh} 4 \pi\left(\mathrm{H} / \lambda_{0}\right)+4 \pi\left(\mathrm{H} / \lambda_{0}\right)}\right],
$$

où $\lambda_{0}$ est donné par la formule classique d'Airy. Cette formule appelle plusieurs remarques. Tout d'abord, on vérifie que l'approximation au second ordre de $\lambda$ pour la houle de Gertsner en profondeur finie est une fonction de la cambrure $2 \mathrm{~A} / \lambda_{0}$, différente de la loi correspondante, valable pour la houle irrotationnelle. Les conclusions de Mme DUbREIL laissaient prévoir ce résultat; de plus, on vérifie que $\lambda-\lambda_{0}$ est une quantité de second ordre en A. Les applications numériques montrent que la correction (62) de la formule d'Airy est, pratiquement, très petite. On voit donc que la houle étudiée ne diffère des ondes classiques (et cela tant en ce qui concerne son profil que la loi de sa dispersion) qu'à partir des éléments dont l'ordre de grandeur est à la limite de la sensibilité des appareils en service dans les laboratoires. Mais il faut bien remarquer que la nature analytique du mouvement étudié dans ce travail est très particulière.

$\S 28$. - A partir des formules résolutives qui précèdent, on vérifie facilement ce résultat, évident à priori, que la houle de Gertsner en profondeur finie est rotationnelle. Cela tient à ce que les approximations du second ordre du vecteur déplacement sont identiques à celles de M. Mrche; on trouve alors aisément, en se limitant au second ordre :

$$
2 \zeta=A^{2} \mu_{0}^{2} \nu \frac{\operatorname{sh} 2 \mu_{0}\left(y_{0}+\mathrm{H}\right)}{\operatorname{sh}^{2} \mu_{0} \mathrm{H}},
$$

formule où $\zeta$ est la composante du vecteur tourbillon suivant la normale au plan du mouvement. Le lecteur pourra contrôler aisément ce résultat, en portant les formules (12), (36) et (41) dans l'équation :

$$
2 \zeta=\frac{\mathrm{D}[(\partial x / \partial t), x\rceil}{\mathrm{D}\left(x_{0}, y_{0}\right)}+\frac{\mathrm{D}\lceil(\partial y / \partial t), y]}{\mathrm{D}\left(x_{0}, y_{0}\right)}
$$

$\S 28$. - Dans un autre travail, nous montrerons que les formules résolutives que nous avons données ci-dessus pour la houle en profondeur finie se réduisent aux formules de Gertsner, lorsqu'on fait tendre $H$ vers l'infini. Ce passage à la limite est un peu moins élémentaire qu'on ne pourrait le penser à priori. Les indications du $\S 9$ laissent prévoir la légère difficulté à surmonter : nous avons construit notre solution au moyen des variables de Miche alors que les formules de Gertsner font intervenir un autre système de paramètres.

\section{VI. - EQuATION CARTESIENNE DE LA SURFACE LIBRE}

$\S 28$. - Au $\S 25$, nous avons présenté les équations explicites de la houle de Gertsner en profondeur finie, exactes au troisième ordre près, sous forme paramétrique du type (1). Cela satisfait l'analyste, mais peut ne pas suffire au technicien, qui a souvent besoin de connaitre l'équation du profil de l'onde sous la forme:

$$
y=y(x, t)
$$

Nous nous proposons, dans ce chapitre, d'expliciter le second membre à l'ordre d'approxition, adopté dans ce travail, près. 
$\S 29$. - Voici la nature de la question. Les formules (12) donnent l'équation de la surface libre sous la forme paramétrique :

$$
\left.\begin{array}{l}
x=x_{0}+\sum_{1}^{3} \mathrm{~A}^{n} \varphi_{n}\left(x_{0}, o, t\right) ; \\
y=\sum_{1}^{3} \mathrm{~A}^{n} \psi_{n}\left(x_{0}, o, t\right),
\end{array}\right\}
$$

les fonctions $\varphi_{n}\left(x_{0}, o, t\right), \Psi_{n}\left(x_{0}, o, t\right)$ étant définies par (36), (41) et les formules récapitulatives du $\S 25$. Le problème revient à inverser la relation $\left(64_{1}\right)$, c'est-à-dire à former :

$$
x_{0}=x_{0}(x, t, \mathrm{~A})
$$

et à porter le résultat dans $\left(64_{2}\right)$. La marche élémentaire que nous suivrons pour effectuer les calculs diffère sur quelques points de détail de celle de Miche.

En premier lieu on voit que, pour A petit, $\left(64_{1}\right)$ est résoluble en $x_{0}$; car, pour A assez petit, on a évidemment :

$$
\frac{\partial x}{\partial x_{0}}=1+\sum_{1}^{3} A^{n} \frac{\partial \varphi_{n}}{\partial x_{0}} \neq 0 .
$$

Il s'ensuit que (64) phe pour $\mathrm{A}=0$, en sorte que :

$$
x_{0}=x+\sum_{1}^{\infty} \mathrm{A}^{n} \Phi_{n}(x, t)
$$

Pour calculer les approximations successives $\Phi_{n}$, on remplacera, dans $\left(64_{1}\right), x_{0}$ par sa valeur (65) et on développera le résultat en série entière, suivant les puissances de $A^{n}$. La relation ainsi obtenue sera une identité en $A$, d'où les équations de définition de $\Phi_{n}$.

En second lieu, notons que, pour évaluer $y(x, t)$ au troisième ordre près en $\mathrm{A}$, il suffit de calculer $\Phi_{1}$ et $\Phi_{2}$ car, en substituant au second membre de $\left(64_{2}\right) x_{0}$ par sa valeur (65), on voit qu'il faut développer $\psi_{1}\left(x_{0}, t\right)$ jusqu'au second ordre, $\psi_{2}\left(x_{0}, t\right)$ jusqu'au premier et remplacer $\psi_{3}\left(x_{0}, t\right)$ par $\psi_{3}(x, t)$. On forme ainsi aisément le développement limité de la cote de la surface libre :

$$
\dot{y}=\mathrm{A} \psi_{1}+\mathrm{A}^{2}\left(\frac{\partial \psi_{1}}{\partial x} \Phi_{1}+\psi_{2}\right)+\mathrm{A}^{3}\left(\frac{\partial \psi_{1}}{\partial x} \Phi_{2}+\frac{1}{2} \frac{\partial^{2} \psi_{1}}{\partial x^{2}} \Phi_{1}^{2}+\Phi_{1} \frac{\partial \psi_{2}}{\partial x}+\psi_{3},\right)
$$

où les valeurs des $\psi_{n}\left(x_{0}, o, t\right)$ et de leurs dérivées sont calculées pour $x_{0}=x$.

$\S 30$. - Passons au calcul des $\Phi_{n}$. Portons dans $\left(64_{1}\right)$ le développement limité :

$$
x_{0}=x+\sum_{1}^{2} \mathrm{~A}^{n} \Phi_{n}
$$

déduit de (65); nous aurons, en suivant la marche esquissée au précédent paragraphe, l'identité valable pour $\mathrm{A}$ assez petit à $\mathrm{A}^{3}$ près :

$$
x \equiv x+\mathrm{A}\left(\Phi_{1}+\varphi_{1}\right)+\mathrm{A}\left\lceil\Phi_{2}+\Phi_{1} \frac{\partial \varphi_{1}}{\partial x}+\varphi_{2}\right]
$$

où les valeurs des $\varphi_{n}\left(x_{0}, o, t\right)$ et de leurs dérivées sont encore calculées pour $x_{n}=x$. D'après ce qu'on a vu, ceci entraîne :

$$
\begin{aligned}
& \Phi_{1}=-\varphi_{1}(x, o, t) ; \\
& \Phi_{2}=\left.\quad \varphi_{1} \frac{\partial \varphi_{1}}{\partial x}\right|_{x_{0}=x}-\varphi_{2}(x, o, t),
\end{aligned}
$$

où $\varphi_{1}$ et $\varphi_{2}$ sont donnés par (36) et (41) respectivement. Portons ces valeurs des $\Phi_{1}$ et $\Phi_{2}$ dans (66). En posant, pour simplifier les écritures :

$$
\begin{aligned}
& y_{2}=\mathbf{A} \psi_{1}+\mathrm{A}^{2}\left[\frac{\partial \psi_{1}}{\partial x} \Phi_{1}+\psi_{2}\right] \\
& \mathrm{Y}_{3}=\frac{\partial \psi_{1}}{\partial x} \Phi_{2}+\frac{1}{2} \frac{\partial 2 \psi_{1}}{\partial x^{2}} \Phi_{1}^{2}+\Phi_{1} \frac{\partial \psi_{2}}{\partial x}+\psi_{3},
\end{aligned}
$$


l'équation de la surface libre s'écrit :

$$
y=y_{2}+\mathrm{A}^{3} \mathbf{Y}_{3} \text {. }
$$

On trouve d'abord [cf. (8), (36), (41) et (67)] :

$$
y_{2}=\mathrm{A} \cos v_{0}+\frac{\mathrm{A}^{2} \mu_{0}}{4} \operatorname{coth}\left(\mu_{0} \mathrm{H}\right)\left[3 \operatorname{coth}^{2}\left(\mu_{0} \mathrm{H}\right)-1\right] \cos 2 v_{0}
$$

C'est l'équation de la surface libre, exacte au second ordre près, donnée par Miche. Nous ne donnerons pas tous les détails des calculs de $Y_{3,}$, définis par (66) et (68) :

$$
\mathbf{Y}_{3}=\psi_{3}-\left(\varphi_{1} \frac{\partial \psi_{2}}{\partial x}+\varphi_{2} \frac{\partial \psi_{1}}{\partial x}\right)+\frac{\partial}{\partial x}\left(\frac{\varphi_{1}^{2}}{2} \frac{\partial \psi_{1}}{\partial x}\right)
$$

On trouve alors successivement $[$ cf. (36)] :

$$
\frac{\partial}{\partial x}\left(\frac{\varphi_{1}^{2}}{2} \frac{\partial \psi_{1}}{\partial x}\right)=\frac{3}{8} \mu_{0}^{2} \operatorname{coth}^{2} q\left(\cos 3 v_{0}-\cos v_{0}\right)
$$

et [cf. (41)]:

$$
-\left(\varphi_{1} \frac{\partial \psi_{2}}{\partial x}+\varphi_{2} \frac{\partial \psi_{1}}{\partial x}\right)=\frac{\mu_{0}^{2}}{16}\left(15 \operatorname{coth}^{2} q+1\right)\left(\operatorname{coth}^{2} q-1\right)\left(\cos 3 v_{0}-\cos v_{0}\right) \text {. }
$$

Enfin, (50), (51) et (61) donnent, si l'on fait $y_{0}=0$ :

$$
c_{33}(o)=(3 / 32) \mu_{0}^{2}\left(\operatorname{coth}^{2} q-1\right)\left(27 \operatorname{coth}^{4} q-42 \operatorname{coth}^{2} q-1\right) .
$$

L'ensemble de ces résultats permet d'écrire :

$\mathrm{Y}_{3}=\frac{\mu_{n}^{2}}{16}\left[\frac{}{4}\left(\operatorname{coth}^{4} q+1\right)\left(3 \operatorname{coth}^{2} q-1\right) \cos 3 v_{0}-\left(15 \operatorname{coth}^{4} q-8 \operatorname{coth}^{2} q-1\right) \cos v_{0}\right]-\frac{\mu_{0}^{\prime \prime} x}{2} \sin v_{0}$

Les formules (68), (69), (70) et (71) donnent l'équation cherchée de la surface libre.

En utilisant la formule (10) et en faisant un changement de paramètre A, pour que le nouveau paramètre a soit l'amplitude du fondamental de l'équation, on peut écrire l'équation de la surface libre sous la forme:

$$
\begin{aligned}
& y=a \cos \mu \mathrm{X}+a^{2}(\mu / 4) \operatorname{coth} \mu \mathrm{H}\left[3 \operatorname{coth}^{2} \mu \mathrm{H}-1\right] \cos 2 \mu \mathrm{X} \\
& +a_{3}\left(3 \mu^{2} / 64\right)\left(3 \operatorname{coth}^{4} \mu \mathrm{H}+1\right)\left(3 \operatorname{coth}^{2} \mu \mathrm{H}-1\right) \cos 3 \mu \mathrm{X} \ldots
\end{aligned}
$$

où :

$$
\begin{aligned}
& \mathrm{X}=x-c t, \\
& \mu=(2 / \lambda)
\end{aligned}
$$

et où $c$ se déduit de $(62)$.

On vérifie ainsi que pour les mêmes valeurs de $a$ et $\lambda$, le profil de la houle que nous avons étudié coïncide avec celui de l'onde irrotationnelle de Stokes jusqu'aux termes du troisième ordre en $a$ inclusivement; mais, d'après (62), la vitesse de propagation des deux houles sera différente.

Ce résultat pouvait être prévu; il constitue, en effet, le cas particulier d'un théorème, encore inédit, de A. Daubert et dont voici l'énoncé :

L'état tourbillonnaire $2 \zeta=f(\psi)$ de l'onde n'influe que sur les termes du troisième ordre en $a$ de l'équation de la surface libre si $f(\psi)$ est du premier ordre en $a$; si $f(\psi)$ est du deuxième ordre, sa valeur n'influe que sur les termes du quatrième ordre de l'équation du profil. Or, d'après les résultats du $\S 27, f(\psi)$ est du second ordre en $\mathrm{A}$, dans le cas de notre onde. Ce fait justifie, a priori, la conclusion de l'étude de la surface libre. 


\section{VII. - REMARQUE SUR UN THÉORĖME DE M. BIESEL}

§ 31. - Dans son mémoire [7], M. Biesel a entre autres, démontré un théorème très intéressant, concernant le transfert en masse dans les houles planes et périodiques. Nous nous proposons de rattacher ce théorème à la théorie de Mme Dubreil et d'en préciser la portée.

Reprenons les notations du chapitre I. Soit $\mathrm{OXY}$, le système d'axes lié à la houle plane; nous savons (cf. § 2) que, relativement à ce repère, le mouvement relatif est permanent et périodique en $\mathrm{X}$, de période $\lambda$. Il suffit donc de considérer le mouvement dans le domaine $\mathrm{D}$, limité par deux verticales $\mathrm{X}=\mathrm{X}_{0}$ et $\mathrm{X}=\mathrm{X}_{0}+\lambda$, la portion de la surface libre comprise entre ces droites et le fond $y=-\mathrm{H}$. Si $\psi(\mathrm{X}, \mathrm{Y})$ est la fonction de courant, les composantes du vecteur vitesse relative de la molécule, qui se trouve au point $\mathrm{X}, \mathrm{Y}$ à l'époque $t$, sont :

$$
-\frac{\partial \psi}{\partial y}, \frac{\partial \psi}{\partial x}
$$

en sorte que le tourbillon $\zeta(\mathrm{X}, \mathrm{Y})$ - le même en mouvement relatif et absolu - vaut :

$$
2 \zeta=(1 / 2) \Delta \psi=f(\psi) ; \quad 0 \leqslant \psi \leqslant q .
$$

Rappelons que $q$ est le débit relatif du liquide; sur le fond $\psi(x,-\mathrm{H}) \equiv 0$; sur la surface libre $\psi(x, y)=q$. Enfin, en vertu de la périodicité en $\mathrm{X}$, on a :

$$
\psi(x+\lambda, y)=\psi(x, y) .
$$

$\S 32$. - Cela étant, nous allons rappeler un résultat de Mme Dubreil (cf. [1], $\S 1$ et $\S 5$ ). Pour fixer les idées, nous orienterons $\mathrm{OX}$ dans le sens de la propagation de l'onde, de célérité $c$ positive. Soit $o x y$, le système d'axes fixes, défini à partir du repère mobile au moyen des formules :

$$
\begin{aligned}
& x=\mathrm{X}+c t \\
& y=\mathrm{Y}
\end{aligned}
$$

Soit A $(x, y)$ un point fixe dans le repère fixe $o x y$; nous supposerons que $y$ est inférieur à la cote du creux de la vague, en sorte que A demeure constamment immergé dans le liquide. D'après le théorème de la composition des vitesses, la composante horizontale $u$ de la vitesse absolue d'une particule liquide qui se trouve en A à l'époque $t$ est donnée par la formule :

$$
u=c-(\partial \psi / \partial y) \text {, }
$$

où $\partial \psi / \partial y$ est calculé en un point, dont les coordonnées relatives $\mathrm{X}, \mathrm{Y}$ sont données, à l'instant $t$, par (73). Envisageons alors un segment vertical A B, de longueur petite, mais fixe, $\partial y$; c'est un segment invariablement lié au repère $o x y$. Le débit moyen absolu $\delta \mathrm{Q}$ du liquide (dont la densité est supposée égale à 1) sera par définition :

$$
\frac{\delta \mathrm{Q}}{\delta y}=\frac{1}{\mathrm{~T}} \int_{t_{0}}^{t_{0}+\mathrm{T}}\left(c-\frac{\partial \psi}{\partial y}\right) d t=w(x, y)
$$

M. BiEsel appelle $w$ la vitesse moyenne du transport de masse en couche profonde; d'après (72) et (73), où, rappelons-le, $x$ et $y$ doivent être regardés comme des quantités fixes, la fonction à intégrer est, d'après (72), périodique, de période $\mathrm{T}$ en $t$, en sorte que sa valeur moyenne est indépendante de $t_{0}$. Pour établir d'autres proprićtés de $w$, il est commode d'effectuer le changement de variables (73). D'après ces formules (*), le point A décrit dans le repère O X Y le segment horizontal $\mathrm{MN}$ :

$$
\mathrm{Y}=y, \mathrm{X}_{0} \leqslant \mathrm{X} \leqslant \mathrm{X}_{0}+\lambda, \mathrm{X}_{0}=x-c t_{0},
$$

intérieur à $\mathrm{D}$ et complètement immergé; de plus :

$$
d \mathrm{X}=-c d t
$$

en sorte que (75) s'écrit :

$$
\begin{array}{r}
w(y)=-\frac{1}{c \mathrm{~T}} \int_{\mathrm{x}_{0}}^{\mathrm{X}_{n-\lambda}}\left(c-\frac{\partial \psi}{\partial \mathrm{Y}}\right) d \mathrm{X} \\
=\frac{1}{\lambda} \int_{\mathrm{X}_{0}}^{\mathrm{x}_{0}+\lambda}\left(c-\frac{\partial \psi}{\partial \mathrm{Y}}\right) d x,
\end{array}
$$

en utilisant, encore une fois, le fait que la valeur moyenne de la fonction $\partial \psi / \partial Y$ de $X$, périodique en $X$, ne dépend pas de $X_{0}$. On voit ainsi directement que $w(Y)$ ne dépend que de la profondeur $\mathrm{Y}$ seule.

La relation (76) a été fréquemment utilisée par Mme DubreiL; nous allons montrer que le théorème de M. Bieses en est un corollaire immédiat.

$\$ 33 . . .-$ En vertu de (72), on a :

$$
\int_{X_{0}}^{x_{0}+\lambda} \frac{\partial^{2} \psi}{d X^{2}} d X=\left(\frac{\partial^{\psi} \psi}{\partial x}\right)_{x=x_{0}}^{x_{0}+\lambda}=0
$$

(*) L'expérience de l'enseignement et les entretiens avec de nombreux ingénieurs nous incitent a penser qu'il y a intérêt à expliciter toutes les transformations élémentaires du texte, 
le chemin d'intégration étant toujours le segment MN. Combinée avec (76), la relation précédente donne :

$$
\begin{aligned}
\frac{d w}{d \mathbf{Y}} & =-\frac{1}{\lambda} \int_{\mathrm{X}_{0}}^{\mathrm{x}_{0}+\lambda} \Delta \psi d \mathrm{X} \\
& =-\frac{1}{2 \lambda} \int_{\mathrm{X}_{0}}^{\mathrm{X}_{0}+\lambda} \zeta(\mathrm{X}, \mathbf{Y}) d x
\end{aligned}
$$

Ainsi, pour toute houle de Mme Dubreil, la demi-valeur moyenne du tourbillon, le long de tout segment $\mathrm{M} \mathrm{N}$, de Iongueur $\lambda$, entièrement immergé, est égale à la dérivée relativement à la profondeur de la vitesse de transport des masses : c'est en cela que consiste le premier théorème de M. Biesel, que cet auteur a justifié par un calcul direct, équivalent au fond, aux raisonnements qui précèdent.

$\S 34$. - D'après (77), on voit que $w(\mathbf{Y}) \equiv 0$ dans le cas d'une houle irrotationnelle. A la suite de M. Biesel, nous nous proposons de démontrer la réciproque. La question est donc de savoir si l'identité :

$$
\frac{d w}{d \mathrm{Y}} \equiv 0,
$$

valable le long de tout segment immergé $\mathrm{MN}$, entraîne :

) $\quad \zeta=0, \quad$ dans D.

L'hypothèse (78) entraîne :

$$
w(\mathbf{Y})=\mathrm{C} \quad \text { dans } \mathrm{D} .
$$

$\mathrm{C}$ étant une constante. On peut, sans restreindre la généralité, prendre $\mathrm{C}=0$; il suffit, en effet, de poser :

pour que :

$$
\psi=\psi_{1}+\frac{C}{\lambda} \mathbf{Y}
$$

$w_{1}(\mathbf{Y})=\frac{1}{\lambda} \int_{\mathrm{X}_{0}}^{\mathrm{x}_{0}+\lambda}\left(c-\frac{\partial \psi_{1}}{d \mathbf{Y}}\right) d \mathrm{X}=0$, dans $\mathrm{D}$.

De même, on peut ajouter à $\psi$ une fonction linéaire de $\mathrm{Y}$ telle que la nouvelle fonction de courant $\psi_{2}$ vérifie la condition :

$$
w_{2}(\mathrm{Y})=-\frac{1}{\lambda} \int_{\mathrm{X}_{0}}^{\mathrm{x}_{0}+\lambda} \frac{\partial \psi_{\mathrm{o}}}{d \mathrm{Y}} d \mathrm{X} \quad \text { dans } \mathrm{D}
$$

Il est clair que :

$$
\frac{d w}{d \mathrm{Y}} \quad \frac{d w_{1}}{\partial \mathrm{Y}}=\frac{d w_{0}}{d \mathrm{Y}}=-\frac{1}{2 \lambda} \int_{\mathrm{X}_{0}}^{\mathrm{X}_{0}+\lambda} \zeta(\mathrm{X}, \mathrm{Y}) d \mathrm{X} \text {, dans } \mathrm{D} \text {. }
$$

puisque l'addition à $\psi$ d'une fonction linéaire de $\mathrm{Y}$ ne change pas $\Delta \psi$; par suite, le tourbillon $\zeta$ demeure inchangé. Par contre, la célérité du mouvement est altérée lorsqu'on modifie la fonction de courant comme il vient d'être dit; et c'est en vue de définir $c$ que Mme Durnerl a insisté sur les propriétés précédentes de $w(y)$. Nous pouvons, en tout état de cause, écrire [cf. (80)]

$$
w(\mathrm{Y}) \equiv 0, \quad \text { dans } \mathrm{D} .
$$

Voici maintenant le raisonnement de M. BiESEL. On peut, sans nuire à la généralité, supposer que $\mathrm{X}=0$ est l'axe de symétrie de $\mathrm{D}$, l'existence d'un tel axe résultant de [1]. Il faut prendre alors $\mathrm{X}_{0}=-(\lambda / 2)$; les bords verticaux $\mathrm{X}_{0}=-(\lambda / 2)$ et $\mathrm{X}_{0}=(\lambda / 2)$ sont aussi des axes de symétrie du mouvement; nous admettrons que le creux de la vague $D$ est situé sur $O \mathrm{Y}$. Chaque ligne de courant $\mathrm{C}(k)$ :

$$
\psi(\mathrm{X}, \mathrm{Y})=k, \quad 0 \leqslant k \leqslant q, \quad k=\mathrm{C}^{\text {te }}
$$

coupe à angle droit chacun des bords verticaux de $\mathrm{D}$ et l'axe $\mathrm{O} Y$, et partage $\mathrm{D}$ en deux domaines simplement connexes. Les résultats de [1] prouvent même qu'on peut résoudre en Y l'équation de $\mathrm{C}(k)$ et l'écrire sous la forme :

$$
\mathrm{F}(\lambda / 2, k) \geqslant \mathrm{Y}=\mathrm{F}(\mathrm{X}, k) \geqslant \mathrm{F}(o, k)
$$

les deux courbes $\mathrm{C}(k)$ et $\mathrm{C}\left(k^{\prime}\right)$ sont dépourvues de point commun, si $k \neq k^{\prime} ; \mathrm{F}(\mathrm{X}, k)$ étant non décroissant pour $0 \leqslant x \leqslant(\lambda / 2)\left({ }^{*}\right)$.

Soit maintenant $\zeta=f(\psi)$. M. BIESEL suppose a priori que sur l'intervalle $0 \leqslant \psi \leqslant q, f(\psi)$ ne peut changer de signe qu'un nombre fini de fois. Appelons : $\psi_{i}, i=1,2 \ldots n$ les zéros de $f(\psi)$, $0<\psi_{1}<\psi_{2} \ldots<\psi_{n}<q=\psi_{n+1}$ où $f(\psi)$ change de signe; $C_{p}$ la ligne de courant $\psi(X, Y)=\psi_{p}$; $-(\lambda / 2) \leqslant \mathrm{X} \leqslant(\lambda / 2) ; p=0,1,2, n+1 ; \mathrm{Y}_{p}$ la cote de l'intersection $\mathrm{A}_{p}$ de $\mathrm{C}_{p}$ avec $\mathrm{O} \mathrm{Y} ; \mathscr{O}_{p}$ le rectangle $-(\lambda / 2) \leqslant \mathrm{X} \leqslant(\lambda / 2), 0 \leqslant \mathrm{Y} \leqslant \mathrm{Y}_{p}$, $p=0,1 \ldots n+1 ; \quad d_{n}$ le domaine limité par $-(\lambda / 2) \leqslant \mathrm{X} \leqslant(\lambda / 2), \mathrm{C}_{p}$ et $\mathrm{C}_{p+1}$. Notons que $\mathrm{C}_{0}$ est le fond, $\mathrm{C}_{n+1}$ la surface libre.

D'après les propriétés du réseau des lignes de courant que l'on vient de rappeler, le rectangle $\mathscr{O}_{p}$ sera contenu dans le domaine :

$$
-(\lambda / 2) \leqslant X \leqslant(\lambda / 2), Y \geqslant-H
$$

et limité supérieurement par $\mathrm{C}_{p}$; rappelons que $\mathrm{C}_{p}$ et l'horizontale $\mathrm{Y}=\mathrm{Y}_{p}$ n'auront en commun que le point $A_{p}$, à moins que $C_{p}$ ne soit un segment horizontal. Des définitions précédentes, il résulte que le tourbillon $\zeta=f(\psi)$ garde un signe constant dans chaque domaine $d_{n}$ (à moins qu'il ne soit nul) et change de signe lorsqu'on passe de $d_{n}$ à $d_{n+1} ; \zeta=0$ sur chaque $\mathrm{C}_{p}$.

Cela étant, on tire de (77) la relation [cf. (81)]:

$$
\int_{0}^{\mathrm{x}_{1}} \frac{d w}{d \mathrm{Y}} d \mathrm{Y}=w(\mathbf{Y})=-\frac{1}{2 \lambda} \iint_{\mathfrak{Q}_{1}} \zeta d \mathrm{X} d \mathbf{Y}=0
$$

(*) Ce dernier résultat est probable; mais, à notre connaissance, il n'a pas encore été démontré en toute rigueur. 
Mais, d'après les hypothèses faites, $\zeta$ ne change pas de signe dans $d_{1}$, donc dans $\mathscr{O}_{1}$. Il s'ensuit :

$$
(1 / 2) \Delta \psi \equiv \zeta \equiv 0 \quad \text { dans } \mathscr{O}_{1}
$$

M. Biesel en conclut directement que l'identité précédente est encore vraie dans tout le domaine $d_{1}$. A notre avis, la justification rigoureuse de ce point essentiel exige les quelques précautions que voici. La démonstration précédente prouve seulement, nous semble-t-il, que $\zeta=0$ dans $\mathscr{O}_{1}$, donc en particulier sur le segment : $-(\lambda / 2) \leqslant \mathrm{X} \leqslant(\lambda / 2), \mathrm{Y}=\mathrm{Y}_{1}$. Comme : $\zeta=f(\psi)$, il s'ensuivrait que le segment précédent serait une ligne $\mathrm{C}_{1}$. Or, il résulte de [1] que par un point de $\mathrm{D}$ il ne passe qu'une ligne de courant. et une seule. Donc, ou bien $\mathrm{C}_{1}$ se réduit à un segment de droite - auquel cas la fonction harmonique $\psi[\mathrm{cf}$. (83)] serait linéaire en $\mathrm{Y}$ (puisque périodique en $\mathrm{X}$, donc bornée dans toute la bande $-\infty \leqslant \mathrm{X} \leqslant \infty, 0 \leqslant \mathrm{Y} \leqslant \mathrm{Y}_{1}$ et constante sur chacun des bords de celle-ci); ou bien $\mathrm{C}_{1}$ n'ést pas rectiligne, auquel cas il résulterait de (83) que $\zeta \equiv 0$ dans $d_{1}$ et $d_{1} \neq \mathscr{O}_{1}$.

Considérons alors le rectangle $\mathcal{O}_{2}$. D'après ce qui précède, $\zeta$ ne change pas de signe dans $\mathscr{O}_{2}$; car $\mathscr{O}_{2}$ se compose, d'une part, de tout le domaine ou d'une partie de $d_{1}$ - où $\zeta$ est nulle et d'une portion de $d_{2}$, d'autre part, où $\zeta$ ne change pas de signe. On peut donc écrire la relation $[\mathrm{cf} .(82)]$ :

$$
\int_{0}^{\mathrm{Y}_{2}} \frac{d w}{d \mathrm{Y}} d \mathrm{Y}=w\left(\mathrm{Y}_{2}\right)=-\frac{1}{2 \lambda} \iint_{\mathfrak{O}_{2}} \zeta d \mathrm{X} d \mathrm{Y}=0
$$

et en déduire, comme ci-dessus, que $\zeta$ est nul danc $\mathcal{O}_{2}$, donc dans $d_{1}+d_{2}$. De proche en proche, on prouvera que $\zeta \equiv 0$ dans tout le domaine $\mathcal{O}$; comme cas particulier, on peut avoir un écoulement où les lignes de courant seraient des segments horizontaux; $\psi(X, Y)$ est alors une fonction harmonique, linéaire de $\mathrm{Y}$ et la surface libre se réduit à un segment de droite.

Ainsi, la réciproque du résultat de $\mathrm{M}$. Biesel est établie (sous la réserve que le nombre de changements de signe soit fini).

$\S 35$. - A lire [7], il semble que M. Biesel lie le résultat qu'il a si simplement et si élégamment démontré, à l'hypothèse faite concernant le nombre de changements de signe de $\zeta=f(\psi)$ sur $0 \leqslant \psi \leqslant q$ qui doit être fini. S'il en était ainsi, le raisonnement en cause n'aurait qu'une portée analytique restreinte. En effet, comme on l'a rappelé au $\S 2$, Mme Dubreru a établi l'exiștence d'une houle périodique correspondant à un choix arbitraire de la fonction $f(\psi)$, astreinte seulement à être continue au sens de HöLdER. Prenons, à titre d'exemple simple, $f(\psi)$ telle que dans le voisinage de $\psi_{1}, 0<\psi_{1}<q$, on ait :

$$
f(\psi)=\sqrt{\left|\psi-\psi_{1}\right|} \sin 1 /\left(\psi-\psi_{1}\right)
$$

On voit que $f\left(\psi_{1}\right)=0$; par suite, dans le voisinage étudié :

$$
\left|f(\psi)-f\left(\psi_{1}\right)\right| \leqslant\left|\psi-\psi_{1}\right|^{1 / 2}
$$

de sorte que $f(\psi)$ est bien hölderienne. Mais cette fonction possède aussi une infinité de zéros simples $\psi_{n}$ donnés par la formule:

$$
\psi_{n}=\psi_{1}+(1 / n \pi)
$$

$n$ étant un entier quelconque. Cet exemnle montre qu'il existe des houles pour lesquelles $f(\psi)$ change de signe une infinité de fois.

On peut étendre la démonstration de M. BIESEL au cas où $f(\psi)$ vérifierait la condition suivante : $f(\psi)$ présente au plus une infinité dénombrable de changements de signe en les points $\psi_{n}$ sur $(o, q)$; aucun point d'accumulation de la suite $\psi_{n}$ ne coïncide avec $\psi=0$. N'ous ne donnerons pas ici ces extensions qui nécessileraient la mise en œuvre de notions, élémentaires d'ailleurs, des théories des ensembles et des fonctions réelles.

Il est moins élémentaire de caractériser d'une manière générale la structure de l'ensemble des valeurs des $\psi$ en lesquels $f(\psi)$, astreinte seulement à être höldérienne, change de signe; puis d'appliquer à de tels ensembles les raisonnements de M. Bresel. Il nous semble préférable d'attaquer la question par un tout autre biais; et c'est ce que nous espérons faire dans une prochaine publication.

\section{BIBLIORAPHIE}

\footnotetext{
1. - Dubrell-J JCOTIN (Mme L.). - Sur la détermination rigoureuse des ondes permanentes périodiques d'ampleur finie.

Thèse de doctorat, Journal de Mathématiques, 13, 1934, pp. 267-291.

2. - Levi-Crvita (T.). - Détermination rigoureuse des ondes permanentes d'ampleur finie. Math. Ann., 93, 1925, pp. 264-280.

3. - Srruurck. - Détermination rigoureuse des ondes irrotationnelles permanentes dans un canal à profondeur finie.

Math. Ann., 1926, pp. 595-610.

4. - Sretensky (L. N.). - Sur une méthode de calcul des ondes d'amplitude finie.
}

Izvestia de l'Académie des Sciences de l'U.R.S.S., tome 5, 1952, pp. 688-698.

5. - Mrche (R.). - Mouvements ondulatoires de la mer. Annales des Ponts et Chaussées, tome 114, 1944, pp. 25-61.

6. - Kravtchenko (J.) et Daubert (A.). - Sur les lois approchées de la houle de Gertsner en profondeur finie.

C.R.A.S., 243, 1956, pp. 2006-2009.

7. - Breser (F.). - Débit. en couche profonde des houles permanentes périodiques.

La Houille Blanche, 1949, pp. 1-4.

8. - Breser (F.). - La Houille Blanche, t. 6, 1951, $\mathrm{n}^{\circ} 3$ pp. $414-416$. 